\title{
Extracellular Vesicle-Mediated Chemoresistance in Oral Squamous Cell Carcinoma
}

\author{
Zhu-Jun Law ${ }^{1}$, Xin Hui Khoo ${ }^{1}$, Pei Tee Lim ${ }^{1}$, Bey Hing Goh ${ }^{2,3}$, Long Chiau Ming ${ }^{4}$, \\ Wai-Leng Lee ${ }^{1 *}$ and Hui Poh Goh ${ }^{4 *}$ \\ ${ }^{1}$ School of Science, Monash University Malaysia, Selangor, Malaysia, ${ }^{2}$ College of Pharmaceutical Sciences, Zhejiang University, \\ Hangzhou, China, ${ }^{3}$ Biofunctional Molecule Exploratory (BMEX) Research Group, School of Pharmacy, Monash University \\ Malaysia, Selangor, Malaysia, ${ }^{4}$ PAP Rashidah Sa'adatul Bolkiah Institute of Health Sciences, Universiti Brunei Darussalam, \\ Gadong, Brunei Darussalam
}

\section{OPEN ACCESS}

Edited by: Miroslaw Kornek, Universität Bonn, Germany

Reviewed by:

Geza Tamas Szabo,

Medical University of Innsbruck, Austria

Sonia Alves Gouvea, Federal University of Espirito Santo,

Brazil

*Correspondence: Wai-Leng Lee lee.wai.leng@monash.edu Hui Poh Goh

pohhui.goh@ubd.edu.bn

Specialty section: This article was submitted to

Molecular Diagnostics and Therapeutics, a section of the journal

Frontiers in Molecular Biosciences

Received: 16 November 2020

Accepted: 12 January 2021

Published: 09 March 2021

Citation:

Law ZJ, Khoo XH, Lim PT, Goh BH, Ming LC, Lee WL and Goh HP (2021)

Extracellular Vesicle-Mediated Chemoresistance in Oral Squamous Cell Carcinoma.

Front. Mol. Biosci. 8:629888. doi: $10.3389 /$ fmolb.2021.629888
Oral Squamous Cell Carcinoma (OSCC) remains a cancer with poor prognosis and high recurrence rate. Even with multimodal treatment options available for OSCC, tumor drug resistance is still a persistent problem, leading to increased tumor invasiveness among OSCC patients. An emerging trend of thought proposes that extracellular vesicles (EVs) play a role in facilitating tumor progression and chemoresistance via signaling between tumor cells. In particular, exosomes and microvesicles are heavily implicated in this process by various studies. Where primary studies into a particular EV-mediated chemoresistance mechanism in OSCC are limited, similar studies on other cancer cell types will be used in the discussion below to provide ideas for a new line of investigation into OSCC chemoresistance. By understanding how EVs are or may be involved in OSCC chemoresistance, novel targeted therapies such as EV inhibition may be an effective alternative to current treatment options in the near future. In this review, the current understandings on OSCC drug mechanisms under the novel context of exosomes and microvesicles were reviewed, including shuttling of miRNA content, drug efflux, alteration of vesicular $\mathrm{pH}$, anti-apoptotic signaling, modulation of DNA damage repair, immunomodulation, epithelial-to-mesenchymal transition and maintenance of tumor by cancer stem cells.

Keywords: oral squamous cell carcinoma, extracellular vesicles, chemotherapy, chemoresistance, oral cancer

\section{INTRODUCTION}

Oral cancer, categorized under head and neck cancer, consists of cancers occurring in the oral cavity. Cancers originated from oral cavity, tongue, lip and mouth accumulatively represents the 8th most common cancer with more than 300,000 cases per annum (D'Cruz et al., 2018; Elaiwy et al., 2020). There were roughly about 65,410 head and neck cancers cases recorded in the United States in 2019, which is accountable for 3.7\% of new cancers (Kim and Kim, 2020). Besides, the incidence and mortality rate for OSCC is regional and its prognostic is still unfavourable (Sim et al., 2019). OSCC is highly mortal despite occurring in a fairly accessible location: the 5-year survival rate stands at only $47-66 \%$ as most oral cancer cases were detected at a late stage of malignancy (Siegel et al., 2017). More than $90 \%$ of cases with oral cancer are classified as squamous cell carcinoma (Leite et al., 2018). Oral squamous cell carcinoma (OSCC) originated from oral keratinocytes. The main risk factors known to cause oral cancer include tobacco smoking and alcohol consumption, while other 
mutagens such as betel quid chewing, diets of low fruit and vegetable content, and infections are known to play a causative role in some oral cancer cases as well (Zain, 2001; Llewellyn et al., 2004; Bunnell et al., 2010; Alnuaimi et al., 2015). Exposure to mutagenic substances can cause spontaneous mutations which induce persistent inflammation in the cells, leading to the development of precancerous lesions. If left untreated, these precancerous lesions (known as leukoplakia and erythroplakia) may further develop into cancerous cells (Belcher et al., 2014).

Current treatments for oral cancer include surgery, chemotherapy, radiotherapy and immunotherapy (Gharat et al., 2016). Depending on the stages of the cancer malignancy, different treatment approaches will be applied. For the early stages, cancer patients are typically treated with either surgery or radiotherapy, and patients with an advanced stage of cancer receive treatment with a combination of surgery and radiation with or without chemotherapy (Belcher et al., 2014). Incorporated multimodality treatment is employed to fully eradicate possible unremoved cancer cells from the first treatment method and has shown to improve the overall survival of OSCC patients (Furness et al., 2011). However, most patients will inevitably experience tumor progression or disease recurrence which usually accompanied with an increase of tumor invasiveness. As reported in many clinical studies, the rate of OSCC recurrence ranged from 18 to $76 \%$, and the majority occurred within 2 years post treatment completion (Myers et al., 2001; Carvalho et al., 2005; Wang et al., 2013). The treatment choices for recurrent OSCC cases are limited; more than $80 \%$ of loco-regional recurrences which happen within the first two years are highly associated with poor prognosis when undergoing salvage surgery (Kernohan et al., 2010). The factors influencing recurrence rate could be due to how advanced the disease is when discovered, development of cells with acquired drug resistance, or the emergence of cell subpopulations genetically refractory to the drugs (Da Silva et al., 2012).

Current research on OSCC drug resistance centers around elimination and inactivation of drug from cancer cells, increased response to DNA damage, reduced apoptosis and increased migratory abilities of cancer cells (Wang et al., 2016).These drug resistance mechanisms are not unique to just OSCC but are shared across different types of cancer cells. An emerging trend of thought is the involvement of extracellular vesicles in mediating these drug resistance mechanism (Khoo et al., 2019). Extracellular vesicles specifically exosomes and microvesicles have been heavily implicated in cancer drug resistance by various studies ( $\mathrm{Yu}$ et al., 2016; Khoo et al., 2019; Steinbichler et al., 2019). The aim of this review is to re-examine OSCC drug resistance mechanisms with the novel lens of extracellular vesicles.

\section{Chemoresistance in OSCC Cells}

Chemoresistance-the resistance of cancer cells to drugs used in chemotherapy-is a major impediment in cancer treatment as it causes long term poor prognosis and increases the chance of metastasis. There are mainly two types of drug resistance in cancer tumors, which are de novo drug resistance and acquired drug resistance. De novo drug resistance is present before drug exposure and selection for drug resistance, while acquired drug resistance, also known as adaptive drug resistance refers to resistance that is developed over time after prolonged exposure to chemotherapy drugs (Hazlehurst and Dalton, 2006). De novo drug resistance arises before drug exposure due to accumulating mutations over time. Some of these mutations may have a selective advantage during chemotherapeutic treatment (Friedman, 2016). Acquired drug resistance has been modeled in tissue culture by chronic exposure to a cytotoxic agent, until a stable drug resistance phenotype is selected. Upon treatment, a pre-existing mutation that carries a selection benefit to the treated tumor cells becomes fixed in the population. The longer the treatment, the higher the likelihood a resistance mutation will be fixed (Friedman, 2016). Moreover, other adaptive responses, such as decreased expression of the therapeutic target and activation of alternative compensatory signaling pathways may arise during treatment, contributing to adaptive resistance (Longley and Johnston, 2005). As such, the intuition that an effective chemotherapeutic drug eliminates the bulk of cancer cells and induces short-term remission may be misleading as the elimination process may effectively select for a chemoresistant subpopulation.

Chemotherapy drugs which are used for the treatment of OSCC include platinum-based drug e.g., like cisplatin and carboplatin, taxanes like paclitaxel and docetaxel, anthracyclines such as adriamycin, epirubicin, pirarubicin, doxorubicin and antimetabolites such as methotrexate and 5fluorouracil (5-FU) (Figure 1). They often work by inducing molecular cascades which result in cell cycle arrest or cell death in cancerous tumors. When a chemotherapy drug directly or indirectly induces damage to DNA, a mechanism known as the DNA damage response (DDR) is activated to coordinate various pathways which result either in DNA repair and cell cycle arrest or apoptosis of damaged cells (Helena Lobo et al., 2007). Chemotherapy drugs such as paclitaxel and docetaxel act by stabilizing microtubules, causing a G2M arrest and later inducing apoptosis (Shah and Schwartz, 2001). On the other hand, platinum-based drugs acts as a DNA intercalating agent and is able to cause DNA damage directly, which leads to the activation of cyclin-dependent kinase inhibitors (CDKIs) and inducing cell cycle arrest in the G2 phase (Sorenson and Eastman, 1988). Anthracyclines such as doxorubicin intercalate between DNA base pairs and inhibits topoisomerase II essential in resolving supercoiling during DNA replication. In addition, antimetabolites such as methotrexate and 5-FU inhibit the action of thymidylate synthase, preventing dTTP production and DNA replication.

Platinum-based chemotherapy drugs which are commonly used in combination with 5-FU are still the usual first-line treatment for OSCC, but the results are far from satisfactory. In advanced OSCC cases, chemotherapy drugs such as methotrexate, paclitaxel and docetaxel are more commonly used either alone or in combination (Specenier and Vermorken, 2010). Despite initial significant results in the 


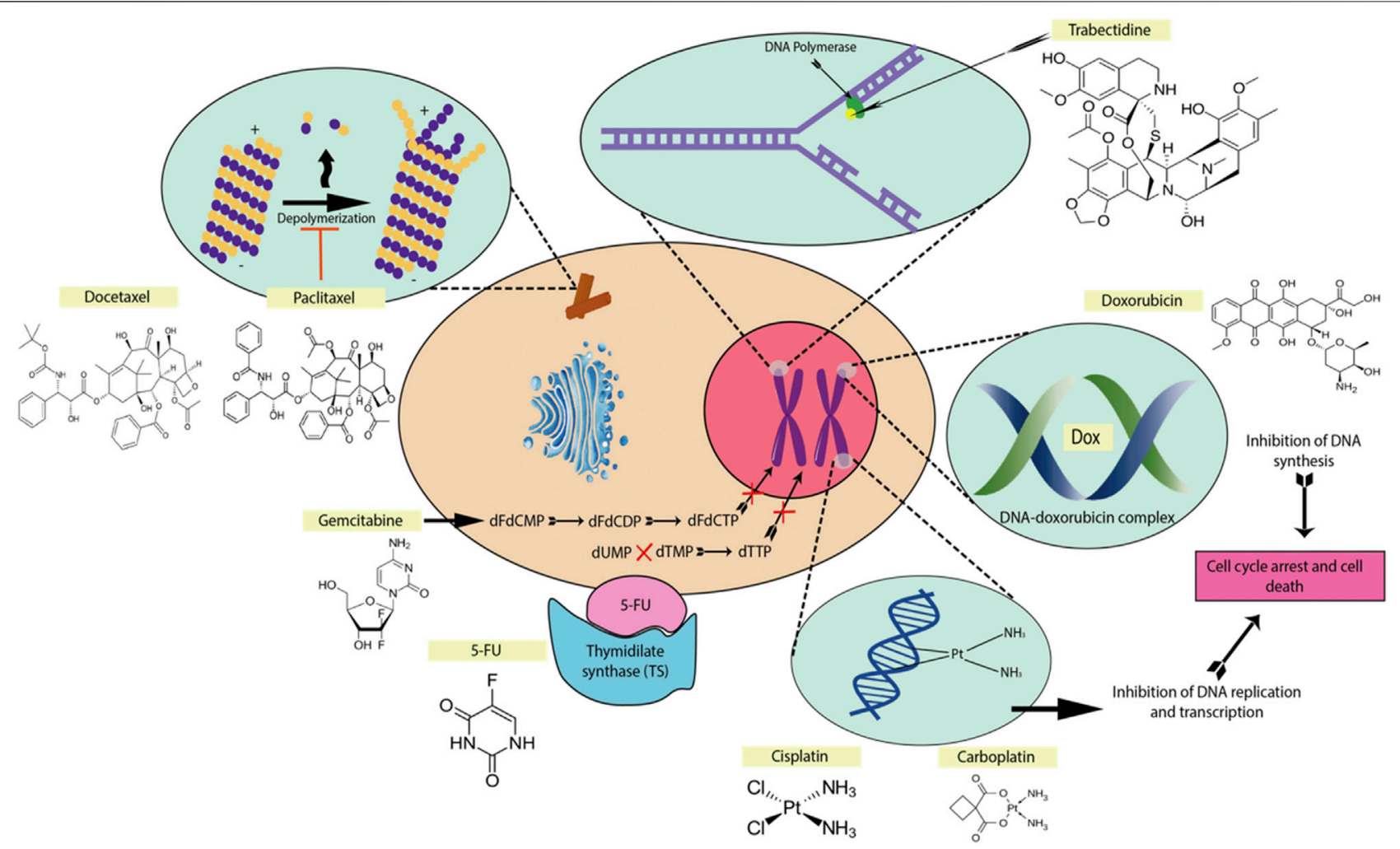

FIGURE 1 | The mechanisms of action of some common chemotherapeutic drugs. The main mechanisms are forming DNA crosslinks (cisplatin, carboplatin), disrupting topoisomerase-II-mediated DNA repair (doxorubicin), promote microtubule polymerization and stabilization (paclitaxel, docetaxel), binding to the minor groove of DNA (trabectedin), inhibiting thymidylate synthase (5-fluorouracil), antimetabolite for pyrimidine nucleoside (gemcitabine) (Larionova et al., 2019).

survivability OSCC patients, these treatments ultimately fail due to the development of chemoresistance.

\section{ROLE OF EXTRACELLULAR VESICLES IN OSCC CHEMORESISTANCE}

Extracellular vesicles (EVs) are lipid-bilayer enclosed vesicles that are naturally released from cells. Over the last decade, intercellular communication mediated by EVs present a new paradigm in which chemoresistance mechanisms should be investigated. Unlike traditional cell-cell signaling via cytokines, chemokines and growth factors (Ahmed and Xiang, 2011), EVs contain cellular cargoes such as miRNAs, lncRNA and proteins that may be endocytosed by other recipient cells. Accumulating evidence points to these cargoes mediating drug resistance properties from cell-to-cell in various cancer types.

EVs can be differentiated into three main categories: exosomes $(30-100 \mathrm{~nm})$, microvesicles (MVs) $(100-1,000 \mathrm{~nm})$, and the more recently discovered 'oncosomes' (1-10 $\mu \mathrm{m}$ ) (Minciacchi et al., 2015). These categories are also differentiated by their mechanism of biogenesis and cellular origins. It is also of importance to note that EV size distinctions are not absolute (i.e. MVs may be $<100 \mathrm{~nm}$ ) and methods to distinguish between exosomes and MVs remain ambiguous from laboratory to laboratory. Since most studies of EV-mediated chemoresistance in OSCC have been conducted on exosomes and MVs, the main focus below will be on these two categories.

\section{Exosomes}

Exosomes are the smallest subset of extracellular vesicles secreted by cells. Their sizes $(30-100 \mathrm{~nm})$ vary depending on the cellular source or sample isolation and preparation methods (Wu et al., 2015). They were first discovered in maturing mammalian reticulocytes during the golden era of electron microscopy. It was observed that maturing reticulocytes contained large sacs filled with small membrane enclosed vesicles of nearly uniform size $(30-100 \mathrm{~nm})$ within their cytoplasm (Johnstone, 2005). In both normal cells and cancer cells, exosomes play a role in the removal of unwanted materials from the cell and communication between cells via the transfer of bioactive molecules packaged into the exosomes. The efflux of exosomes is one way of eliminating cell waste products-the other way being lysosomal degradation (autophagy) (Baixauli et al., 2014).

Exosomes originate from the early endosome which accumulates intraluminal vesicles (ILVs) formed through the inward budding of its membrane (Colombo et al., 2014). The endosomes are referred to as multivesicular bodies (MVBs) due to the multiple ILVs contained in the endosomes. During the inward 


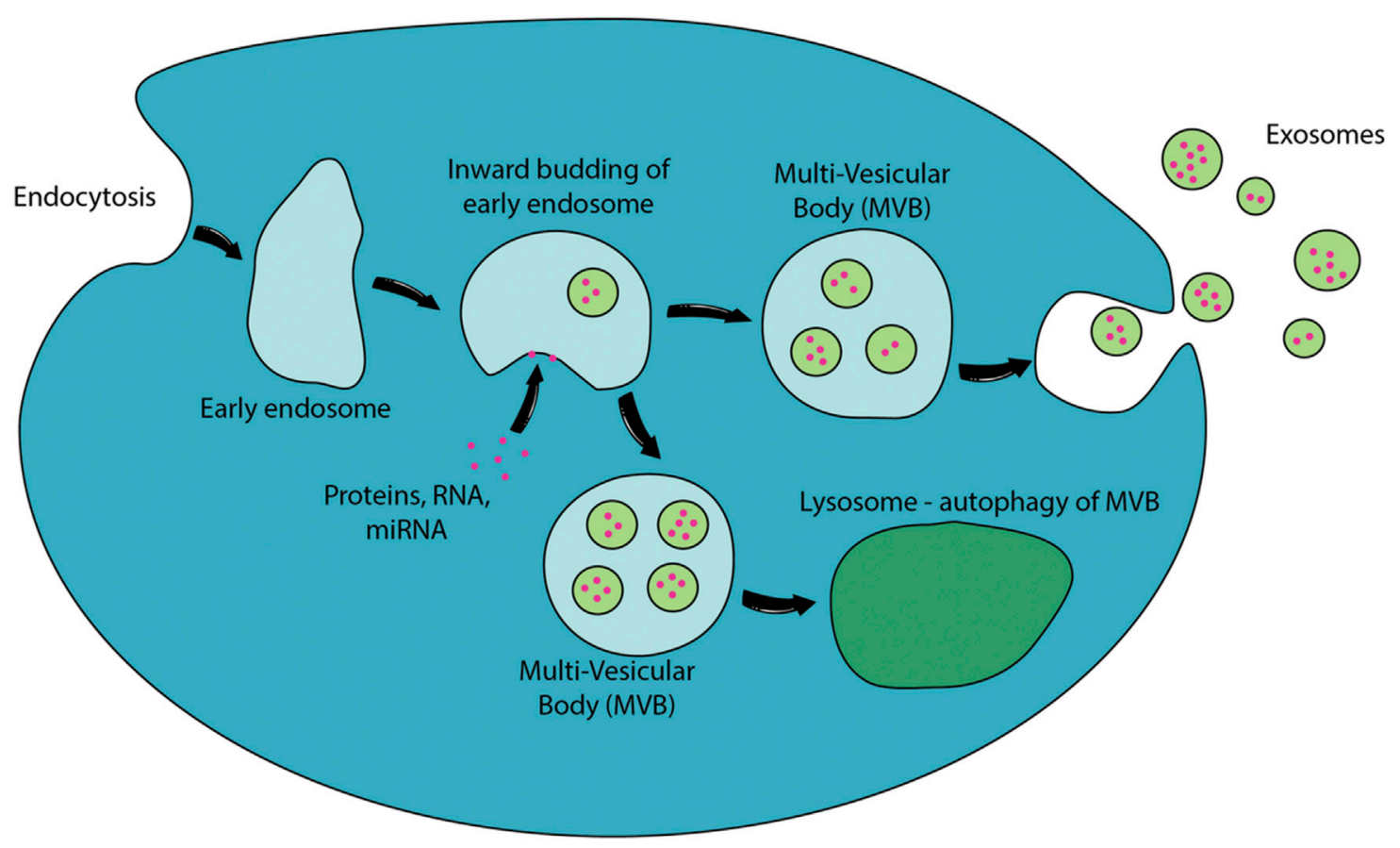

FIGURE 2 |Biogenesis of exosomes. EVs are released to the extracellular environment through direct outward budding or "pinching" of the cellular membranes. Proteins, RNA, miRNA or other potential exosomal contents will be packed into early endosomes. The inward budding of the early endosomes to form intraluminal vesicles and the fusion of multi-vesicular body (MVB) with plasma membrane releases exosomes to the extracellular environment. MVB could also fuse with lysosomes which will degrade their internal contents through autophagy (Raposo and Stoorvogel, 2013).

budding of the early endosome, proteins, lipids and RNAs are selectively sorted into ILVs. The MVBs containing loaded ILVs are either fused with lysosome for degradation or fuse with the plasma membrane for secretion-the secreted ILVs are referred to as exosomes (Colombo et al., 2014). The overall biogenesis of exosomes is presented in Figure 2.

This entire process can either be driven by the endosomal sorting complexes required for transport (ESCRT) or by other ESCRT-independent mechanisms such as neutral sphingomyelinase-dependent ceramide formation. Exosomes secreted from one cell can fuse with the plasma membrane of another cell and can be internalized through endocytosis or pinocytosis, therefore, transferring bioactive molecules from one cell to another (Théry et al., 2002). The exosomal contents that are transferred to other cells can affect the function of the recipient cell. For example, the transfer of exosomal RNA from antigen-presenting dendritic cells can stimulate proliferation in T lymphocytes (Segura et al., 2005). In cancer cells, exosomes play important roles in tumorigenesis, growth, progression, metastasis and drug resistance (Liu et al., 2017).

\section{Exosome-Mediated Chemoresistance}

Exosomes play many important roles in enabling drug resistance in cancer cells through multiple mechanisms such as shuttling of miRNA, drug efflux, anti-apoptotic signaling etc. In order to understand the effect of exosomes in both donor and recipient cells, it is imperative to investigate the exosomal cargo being shuttled between tumor cells.

\section{Exosomal Content (MiRNAs)}

Due to the exosome's ability to transfer diverse loads of proteins, lipids and nucleic acids between cells, they have been dubbed as 'Trojan horses' (Gould et al., 2003). Exosomes secreted by cancer cells contain various functional nucleic acids such as circular DNA, mRNA, microRNAs (miRNAs), long noncoding RNAs (lncRNAs), transfer RNAs (tRNAs), small nucleolar RNAs (snoRNAs), ribosomal RNAs (rRNAs) and small nuclear RNAs (snRNAs) (Gajos-Michniewicz et al., 2014). DNA fragments and mutated mRNA transcripts are involved in the progression and angiogenesis of primary and metastatic cancers (Skog et al., 2009; Dvořáková et al., 2013). Cancer-derived exosomes show specific miRNA and mRNA expression profiles which vary according to the type of cancer (Ming-Hui et al., 2015; Yousafzai et al., 2018).

There is a significant body of evidence supporting the theory that exosomes, in their capacity to act as agents of intercellular communication, disperse the characteristic of drug resistance between tumor cells by delivering miRNAs (Zhang and Wang, 2017). MicroRNAs are small single-stranded highly conserved non-coding RNAs of 20-25 nucleotide length that are involved in post-transcriptional regulation of gene expression. The main function of miRNA is to base-pair with target mRNA to negatively regulate gene expression (Bartel, 2004; Macfarlane 
TABLE 1 | Roles of miRNA in regulation of drug resistance of OSCC.

\begin{tabular}{|c|c|c|c|}
\hline MicroRNAs & OScC Cell lines & Effect & References \\
\hline \multirow[t]{3}{*}{$\operatorname{miR}-21$} & Tca8113/DDP & Regulation of STAT3 expression & (Xuan et al., 2014) \\
\hline & HSC-3-R & Confers cisplatin resistance by targeting PTEN and PDCD4 & (Liu et al., 2017)* \\
\hline & CAL27 & Reprogram monocytes via NF- $\kappa$ B pathway & (Momen-Heravi and Bala, 2018)* \\
\hline \multirow[t]{2}{*}{$\operatorname{miR}-24$} & CAL27, HSC6 & Reduce PTEN expression & (Zheng et al., 2015) \\
\hline & SCC25, HSC6 & Binds to PER1 and induces cell proliferation and cell cycle progression & $($ He et al., 2020)* \\
\hline $\operatorname{miR}-29 a-3 p$ & SCC9 and CAL27 & Promote M2 subtype macrophage polarisation & (Cai et al., 2019)* \\
\hline miR-130b, miR-134, mi491, mi-149 & $\begin{array}{l}\text { SCC131/R, } \\
\text { SCC084/R }\end{array}$ & Modulation of EMT and metastasis & (Ruma Dey et al., 2016) \\
\hline miR-155 & SCC131 & Downregulates FOXO3 and promotes EMT phenotype & (Kirave et al., 2020)* \\
\hline$m i R-221$ & Tca8113, UM2 & Reduce TIMP3 expression & (Chen et al., 2016) \\
\hline miR-222 & UM1 & Reduce PUMA expression & (Jiang et al., 2014) \\
\hline $\operatorname{miR}-1246$ & SAS, GNM & Enhancing cancer stemness and chemoresistance via targeting CCNG2 & (Shih-Shen et al., 2018) \\
\hline
\end{tabular}

STAT3: Signal transducer and activator of transcription 3; PTEN: Phosphatase and tensin homolog; PDCD4: Programmed cell death protein 4; NF- $\kappa B$ : Nuclear factor- $\kappa B$; EMT: EpithelialMesenchymal Transition; TIMP3: Tissue inhibitor of metalloproteinase 3; PUMA: p53 activates transcription of p53-upregulated modulator of apoptosis; CCNG2: Cyclin-G2.

'*' Represents published literature which demonstrated that the respective miRNA was present in OSCC-derived exosomes.

and Murphy, 2010). Regulated by cofactors, miRNAs also play a role in the activation of mRNA translation to regulate protein levels (Saraiya et al., 2013). Aside from that, they play a major role in controlling various activities in cells, including cell differentiation, proliferation, stress response, metabolism, cell cycle, apoptosis, and angiogenesis thus playing roles in the regulation of many diseases, including cancers (Jiang et al., 2009). Table 1 outlines various miRNAs that are implicated in OSCC chemoresistance along with its cellular effects.

A particular miRNA which has been reported widely in modulating cancer drug resistance is miR-21. Although the precise mechanism of action of miR-21 in OSCC has yet to be fully described, miR-21 is regarded as a key oncogenic factor that is associated with poor cancer prognosis and are highly expressed in tissues and blood of OSCC patients when compared to healthy tissues and blood control (Chang et al., 2008; Wong et al., 2008; Ren et al., 2014). MiR-21 was shown to be involved in the regulation of drug resistance in various types of cancers including renal cancer, breast cancer, leukemia cancers, glioblastoma, gastric cancer and many other cancer cell lines (Hong et al., 2013; Yang et al., 2013; Gaudelot et al., 2017). It has been confirmed that miR-21 induces chemoresistance by targeting PDCD4 and PTEN, two tumor suppressor genes (Wei et al., 2016; Liu et al., 2017). PDCD4 is involved in apoptosis and inhibits tumor progression by acting on eIF4A and eIF4G, suppressing mRNA translation (Liu et al., 2017) whereas PTEN diminishes the P13K/Akt/mTOR signaling pathway which results in tumor growth. It was also shown in the study by Liu et al. that (previously chemosensitive) OSCC cells developed resistance after being exposed to exosomes derived from resistant OSCC cells (which contained miR-21) (Liu et al., 2017).

The mechanism of miR-21 in OSCC drug resistance may also be through the regulation of STAT3, a transcription factor activated by IL-6. MiR-21 is known to affect STAT3 to promote tumor cell survival, induce anti-apoptotic effect, and also aid in cell cycle progression and angiogenesis (Iliopoulos et al., 2010; Xuan et al., 2014). The suppression of STAT3 with a specific inhibitor was shown to downregulate miR-21 in OSCC cells and sensitized cisplatin-resistant OSCC cells to cisplatin treatment (Xuan et al., 2014). This result supports the role of miR-21 in moderating cisplatin resistance in OSCC and suggests that STAT/miR-21 pathway can serve as a potential therapeutic target to develop chemosensitivity in cisplatin-resistant OSCC cells.

MiR-24 is another highly upregulated miRNA in OSCC tissues. It regulates a myriad of cellular activities and was reported to induce cell survival and cisplatin resistance in tongue squamous cell carcinoma when up-regulated (Zheng et al., 2015). Frequent dysregulation of miR-24 was observed in OSCC cells; upon further analysis, it was proven that miR-24 directly inhibits PTEN (a tumor suppressor gene) expression, resulting in the activation of the PI3K/Akt cell survival pathway and promoting cell cycle progression (Zheng et al., 2015). Lossof-function mutations of PTEN are known to drive the resistance of cancer cells to many anticancer drugs (Dillon and Miller, 2014). Knockdown of miR-24 was shown to restore PTEN expression and resensitize cisplatin-resistant OSCC cells to cisplatin treatment (Zheng et al., 2015). Other targets of miR-24 besides PTEN include DND1 (regulates CDKN1B suppression which enhanced proliferation and reduced apoptosis in toungue SCC cells) (Liu et al., 2010), and more recently discovered PER1 (enhances cell proliferation and cell cycle progression of OSCC cells) (He et al., 2020). It is interesting to note that $\mathrm{He}$ et al., also proven that miR-24 is a cargo of salivary exosomes derived from OSCC patients. The increased cell proliferation and reduced apoptosis mediated by miR-24 counteracts against chemotherapeutic drug mechanisms such as cell cycle inhibition and DNA damage-induced apoptosis. Interestingly, miR-24 also have anti-tumour characteristics in other cancer types via FOXM1 which suppresses bladder cancer cell proliferation (Inoguchi et al., 2014) and LPAAT which suppresses osteosarcoma cell proliferation (Song et al., 2013). The diverse binding targets of miR-24 in different cancer types prompts further investigation to elucidate the complete pathways that miR-24 is involved in OSCC. This is crucial to increase the feasibility of proposed strategies that therapeutically targets miRNA in OSCC tissues. Without sufficient information of miRNA binding targets, it becomes 
difficult to predict cellular effects from upregulating or downregulating miRNA in OSCC tissues.

The discovery of miRNA involvement in chemoresistance is still novel and studies relating to OSCC are minimal at the moment. Moreover, one miRNA may target various tumorrelated gene transcripts and the activity of one gene transcript may be regulated by multiple miRNAs. Thus, it is challenging to design therapeutic strategies which can efficiently eliminate drug resistance via a single miRNA (Hong et al., 2013). There are recent studies detailing the discovery of 'master miRNAs' which target multiple drug resistance pathways (Manabu et al., 2017). This may be an alternative solution in treating OSCC drug resistance and requires further investigation. Aside from that, studies that prove the above discussed miRNAs to be present in OSCC-derived exosomes are limited. It is important to note that two populations of extracellular miRNAs exist in biological fluids-one found in vesicles such as exosomes and microvesicles; the other is associated with proteins such as Argonaute (AGO) (Turchinovich et al, 2011). There is a significant number of studies that do not investigate the origins of their respective OSCC-associated miRNAs. Therefore, the role of exosomes in conferring chemoresistance traits via these miRNAs have to be viewed with skepticism.

\section{Drug Efflux}

Multidrug resistance (MDR) is a phenotype in which cancer cells exhibit simultaneous resistance to multiple chemotherapeutic drugs. Overexpression of drug efflux transporters of the ATP binding cassette $(\mathrm{ABC})$ transporter family such as P-glycoprotein (P-gp) and copper efflux transporters gives rise to the MDR phenotype in tumor cells (Yoshizawa et al., 2007; Pérez-Sayáns et al., 2010). Although yet to be proven in OSCC cell lines, exosomes from resistant tumor cells can transfer $\mathrm{P}$-gp to chemosensitive cells and confer chemoresistance. Lv et al. (2014) demonstrated that P-gp expression in sensitive MCF-7 breast cancer cells were higher after incubation with exosomes isolated from docetaxel-resistant MCF-7 cells (Lv et al., 2014). However, expression analysis of P-gp in OSCC cells via RT-PCR revealed that only $18 \%$ of OSCC cases are P-gp-positive (Friedrich et al., 2004). P-gp expression in other tumor types such as salivary gland adenocarcinoma and colon carcinoma are markedly higher than in OSCC (Uematsu et al., 2001; Friedrich et al., 2004). Nevertheless, P-gp levels in recurrent OSCCs are higher compared to normal mucosa with lesions at different stages of tumorigenesis (Jain et al., 1997). Aside from P-gp, Multidrug Resistance Associated Protein 1 (MRP1) is also linked to the MDR phenotype as well. MRP1 expression levels measured via RT-PCR and immunohistochemistry revealed that 20-30\% of OSCC cases have higher than normal expression (Tsuzuki et al., 1998; Uematsu et al., 2001). These results show that the set of proteins giving rise to the MDR phenotype in OSCC cells is different compared to other tumor types.

Platinum-based antitumor drugs such as cisplatin and carboplatin are the mainstream chemotherapy drugs used clinically. Ctr1, the major copper influx transporter has been demonstrated to transport cisplatin and other analogues such as carboplatin and oxaliplatin. More evidence of two other copper efflux transporters-ATP7A and ATP7B are also accumulating (Komatsu et al., 2000; Kuo et al., 2007; Yoshizawa et al., 2007). Mechanisms for transporting platinum drugs were not known until evidence of it related to copper homeostasis began to build. Many cell lines that exhibited resistance to platinum drugs also showed cross-resistance to copper drugs and vice versa (Safaei et al., 2005). However, enhanced cisplatin intake via increased expression of copper influx transporter, Ctr1 does not sensitize tumor cells to cisplatin nor does it increase cisplatin-DNA adduct formation (Beretta et al., 2004; Holzer et al., 2004). One plausible reason is that Ctr1 does not deliver cisplatin in a way that allows it to access key intracellular cytotoxic targets. The two copper efflux transporters-ATP7A and ATP7B are located in the trans-Golgi network (TGN) and are involved in copper-stimulated trafficking (Didonato and Sarkar, 1997; Camakaris et al., 1999; Prohaska and Gybina, 2004). Under conditions of excess copper in the cytoplasm, ATP7A and ATP7B are transported from the TGN to the plasma membrane to facilitate copper efflux until copper levels return to normal homeostatic ranges (Hellman and Gitlin, 2002). It is not known if cisplatin levels affect the normal transportation of ATP7A and ATP7B from the TGN to the plasma membrane. Moreover, the vesicular transport pathways between TGN and endosomes are complicated and it is not known if ATP7A/B is transported to the endosomal membrane from the TGN in OSCC cells (Progida and Bakke, 2016).

Another school of thought supports the idea that cisplatin efflux via ATP7A/B involves lysosomal exocytosis (Petruzzelli and Polishchuk, 2019). ATP7A/B residing in the membrane of endo-lysosome (formed via fusion of endosome and lysosome) facilitates the influx of cisplatin into the lumen. Subsequent fusion of the endo-lysosome with the cell membrane releases cisplatin into the extracellular environment. Safaei et al. (2005) demonstrated that cisplatin resistant ovarian carcinoma cells have a lesser endo-lysosome volume and expression of lysosome-associated membrane proteins (LAMP1 and LAMP2), implying a marked increase in lysosomal exocytosis to expel cisplatin (Safaei et al., 2005). However, Safaei et al. (2005) also discovered that exosomes released from the resistant cells contain 2.6-fold more platinum than those released from sensitive cells (Safaei et al., 2005). An alternate mechanism of cisplatin efflux via lysosomal exocytosis involves intraluminal vesicles (ILVs). Transmembrane proteins such as ATP7A/B residing in the membrane of endo-lysosome can end up in new ILVs formed via budding of the endo-lysosomal membrane. Cisplatin are bound to the metal binding sites (MBSs) of ATP7A/B on the endo-lysosomal membrane which will be incorporated into the ILV membrane. The cisplatin will then be transported out from the cells when the ILVs are released as exosomes. Figure 3 below outlines both mechanisms of drug efflux involving ATP7B. Safaei et al. (2005) results support the latter mechanism as they reported a marked increase in exosomal levels of putative cisplatin transporters, mainly ATP7A and ATP7B (Safaei et al., 2005). Such a mechanism does not require the transport of cisplatin across a membrane, but rather requires cisplatin to be bound to the MBSs of ATP7A/B. Hence, it is likely a less energy-consuming mechanism of drug efflux. 

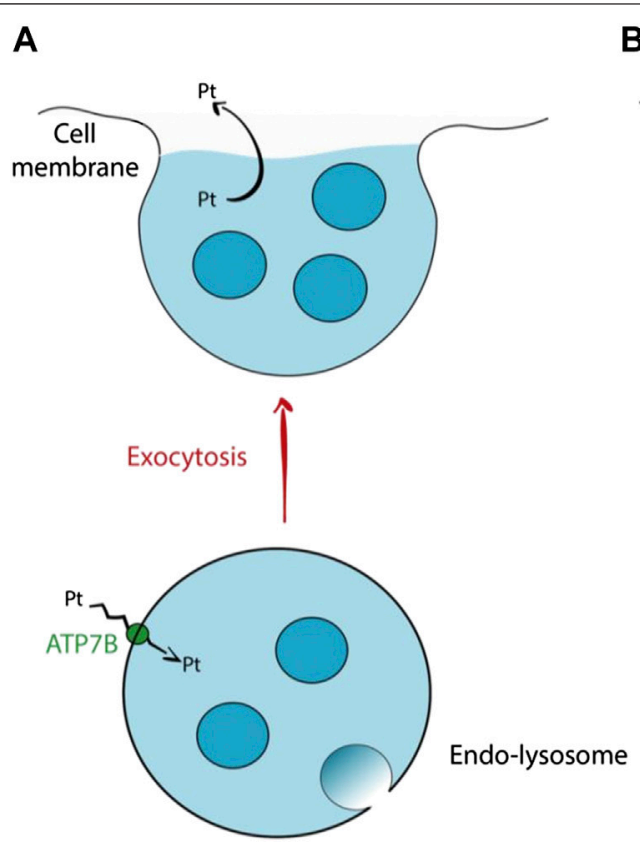

B
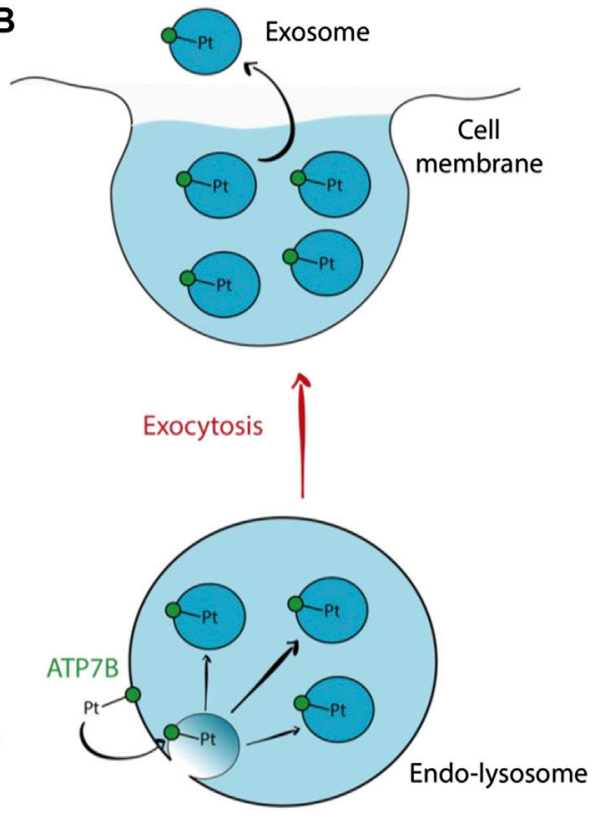

FIGURE 3 | Hypothetical mechanisms of cisplatin efflux via lysosomal exocytosis involving ATP7B (A) ATP7B residing in the endo-lysosomal membrane transports cisplatin across the membrane into the endo-lysosomal lumen. Subsequent fusion of endo-lysosome with the cell membrane releases the cisplatin along with the ILVs. This proposed mechanism does not involve exosomes for cisplatin efflux. (B) Cisplatin is bound to ATP7B via the MBS. The ATP7B will then be incorporated into the membrane of a new ILV formed via budding of the endo-lysosomal membrane. The cisplatin will then follow the ILVs out from the cell when they are released as exosomes (Petruzzelli and Polishchuk, 2019).

Although results from Safaei et al. (2005) have yet to be proven true in OSCC cells, there is already evidence pointing toward ATP7B as a key contributor to cisplatin-resistance in OSCC cells (Safaei et al., 2005). Yoshizawa et al. (2007) demonstrated that resistant OSCC cell lines, namely HSC-4-R, OSC-19-R and HOC313-R all have higher expression of ATP7B (Yoshizawa et al., 2007). Transfection of ATP7B siRNA in OSC-19-R cells resensitized the cells to cisplatin by a factor of 10.6 (Yoshizawa et al., 2007). These results were not observed in ATP7A and Ctr1, pointing to a bigger role played by ATP7B in the context of OSCC cisplatin resistance. It is plausible that cisplatin efflux in OSCC cells may follow the lysosomal exocytotic pathway. However, experiments to measure the volume of lysosome in cisplatinresistant and sensitive OSCC cells are needed to prove this theory. Furthermore, vesicular transport of ATP7B from TGN to endolysosome in OSCC cells remains unproven.

\section{Alteration of Vesicular $\mathrm{pH}$}

The difference in $\mathrm{pH}$ between intracellular and extracellular $\mathrm{pH}$ plays an important role in the transport of chemotherapy drugs. Under normal circumstances, extracellular $\mathrm{pH}$ in OSCC is significantly more acidic than in normal tissue (Perez-Sayans et al., 2009). The acidic environment disrupts the absorption of chemotherapy drugs (Griffiths, 1991; Negendank, 1992). Studies conducted by Becelli et al. (2007) found that reversed $\mathrm{pH}$ gradient is an intrinsic tumor phenotype giving rise to drug resistance in oral cancer (Becelli et al., 2007). The intracellular and extracellular $\mathrm{pH}$ of oral cancer tumor specimens were found to be lower than normal tissues (Becelli et al., 2007).

It has been widely accepted that the changes in $\mathrm{pH}$ are mediated by vacuolar-ATPases (V-ATPases). V-ATPase is a multi-subunit ATP driven proton pump that regulates the intracellular and extracellular pH (Pamarthy et al., 2018). Aside from regulating the acidity of the tumor microenvironment from the plasma membrane (Perez-Sayans et al., 2009), V-ATPases are thought to play a role in vesicular acidification. Martínez-Zaguilán et al. was unable to trace $\mathrm{V}$-ATPases in the plasma membrane via immunohistochemical analysis and concluded that V-ATPases are not statistically resident in the cell membrane of MCF-7 breast carcinoma cells (Martinez-Zaguilan et al., 1999). Resistant MCF7 cells showed increased V-ATPase activity as they were capable of recovering from an acid load without anion exchangers (Martinez-Zaguilan et al., 1999). The failure to localize $\mathrm{V}$-ATPase, coupled with the presence of V-ATPase activity suggests that the measured activity of V-ATPase might be the consequence of "rapid endomembrane turnover". Thus, it can be hypothesized that V-ATPase resides more extensively on vesicle membrane rather than statically in the plasma membrane. This hypothesis is yet to be proven experimentally in OSCC cells. However, it suffices to note that V-ATPase is commonly localized in vesicular membranes of cancerous cells.

Raghunand et al. (1999) postulated that turnover of acidic vesicles is an important factor of chemoresistant cells, particularly 
those that do not overexpress P-gp efflux pumps. They have developed a computational model that accounts for various resistance mechanisms to weakly basic drugs. They found that a combination of mechanisms including active transport of drugs into endosomes, increased endosomal turnover, decreased endosomal $\mathrm{pH}$ and increased cell membrane $\mathrm{pH}$ gradient would yield a drug-resistant phenotype. An example of cells which show such pattern is MCF-7 cells that are chemoresistant can lower cytosolic concentrations of weak base drug such as mitoxantrone (Raghunand et al., 1999).

The regulation of cellular $\mathrm{pH}$ on drug resistance is further proven by another study which discovered that upregulated proton pump gene expression in cisplatin-resistant cell lines which resulted in intracellular alkalization and elevated intracellular $\mathrm{pH}$ in the cells. As DNA-cisplatin adducts are more easily formed under acidic conditions, the increase in the cellular $\mathrm{pH}$ could attenuate its cytotoxicity effect and induce drug resistance, and this was shown when proton pump inhibitor synergistically potentiated the cisplatin cytoxicity (Murakami et al., 2001). Whether or not V-ATPases are overexpressed in endosomal membrane or ILVs was not investigated by Murakami et al. (2001). Studies were conducted to investigate the effect of V-ATPase inhibitors on sensitizing chemoresistant cells (Murakami et al., 2001; Kiyoshima et al., 2013). The treatment resulted in lower acidification of vesicular organelles (detected using acridine orange stain) and increased apoptosis in three of four OSCC cell lines, indicating that vesicular acidification is an important survival mechanism of OSCC cells (Kiyoshima et al., 2013). This further points to the possibility of improving cytotoxicity of drugs such as cisplatin by combining it with proton pump inhibitors. However, it is not well understood if alkalization of vesicles will affect uptake or efflux of chemotherapy drugs.

\section{Anti-Apoptotic Signaling}

Apoptosis is one form of programmed cell death initiated by apoptotic proteins such as caspases, Bcl-2-associated proteins and cytochrome C. Malignant tumors are often marked with the overexpression of anti-apoptotic proteins or signals. Current molecular markers that hold prognostic and predictive values are still incapable of resolving the heterogeneity and complexity of OSCC (Chung et al., 2004). Molecular profiling by gene arrays, tissue microarrays, immunohistochemistry profiling and cluster analysis have been the standard of molecular characterization of tumors.

A study conducted a molecular profiling of 171 OSCC cases via immunohistochemical analysis and ordered them by hierarchical clustering and the expression profiles of 23 antiapoptotic proteins were investigated. Despite being able to divide the OSCC cases into two groups-apoptotic and anti-apoptotic, there was no association between these groups with clinical and pathological characteristics such as the overall survival of patients. Thus, there is a knowledge gap in connecting apoptosis and cancer prognosis that needs to be filled. However, the analysis did showed a group of pro-apoptotic proteins that are more prominent and could be use as targeted therapies (Coutinho-Camillo et al., 2017). The complexity of apoptosis induction and the influence of individual proteins in the apoptotic network needs more elucidation and experimental verification in order to account for the heterogeneity of OSCC tumors.

Notwithstanding the current lack of understanding between apoptosis and cancer survival, apoptotic inhibition is still a chemoresistance trait that needs to be studied in depth. It has been suggested that exosomes can promote anti-apoptotic signals in both donor and recipient cells. For instance, Caspase-3, a protein known as an executioner caspase is responsible for cleaving cellular substrates that are vital to cell survival, leading to membrane blebbing and disruption of cytoskeletal functions. The release of caspase- 3 containing membrane vesicles is thought to facilitate removal of apoptotic proteins in order to promote cell survival (Böing et al., 2013).

Exosomes can transduce anti-apoptotic signals to recipient tumor cells via different mechanisms. They can stimulate recipient cells via signal transduction facilitated by surface receptors to activate anti-apoptotic pathways (Xu et al., 1998; Roccaro et al., 2013). Exosomes can transfer receptors such as CD41 to target cells to trigger integrin-mediated inhibition of apoptosis by preventing cell detachment from the extracellular matrix (Boudreau et al., 1996; Wang et al., 2014). Besides that, exosomes can also transfer transcriptional factors that can induce activation of anti-apoptotic pathways (Wang et al., 2014).

As discussed above, miRNA plays an important role in mediating various signaling pathways between tumor cells as a major exosomal cargo. One of the signaling pathways include anti-apoptosis. Guo et al. showed that exosomes derived from cancer-associated fibroblasts (CAF) confers cisplatin resistance to head and neck squamous cell carcinoma (HNSCC) via exosomal miR-196a (Guo et al., 2019). HNSCC cells (CAL27 and HN4) grown on CAF conditioned media (CAF-CM) promoted cisplatin resistance and cell proliferation compared to media conditioned from chemoresistant HN4 cells (Guo et al., 2019). Physical removal of exosomes from CAF-CM by ultracentrifugation and blocking exosome release via the inhibitors GW4869 reduced the ability of CAF-CM to promote HNSCC cell survival (Guo et al., 2019). With exosomes proven as the mode of delivery for cisplatin-resistance promoting factors, miRNA array analysis of cisplatin treated CAF-derived exosomes was carried out. The test revealed a marked increase in miR-196a content (Guo et al., 2019). Subsequent MTT and colony formation assays showed that miR-196a overexpression enhanced the growth of CAL27 and HN4 HNSCC cells transfected with miR-196a (Guo et al., 2019). The target genes that interact with miR-196a were identified via miRecords algorithm to be CDKN1B and ING5. RT-PCR revealed that both genes were inhibited by miR-196a overexpression (Guo et al., 2019). ING5 is a tumor suppressor protein that inhibits cell growth and apoptosis (Gao and Han, 2018), whereas CDKN1B is a cell cycle inhibitor that slows down the progression of cell division (Cusan et al., 2018). Although the study was conducted on HNSCC cells and not specifically OSCC, it is worth noting the effects of CAF-derived exosomes on squamous cell carcinomas. Moreover, it provides further evidence of the tumor microenvironment playing an important role in 


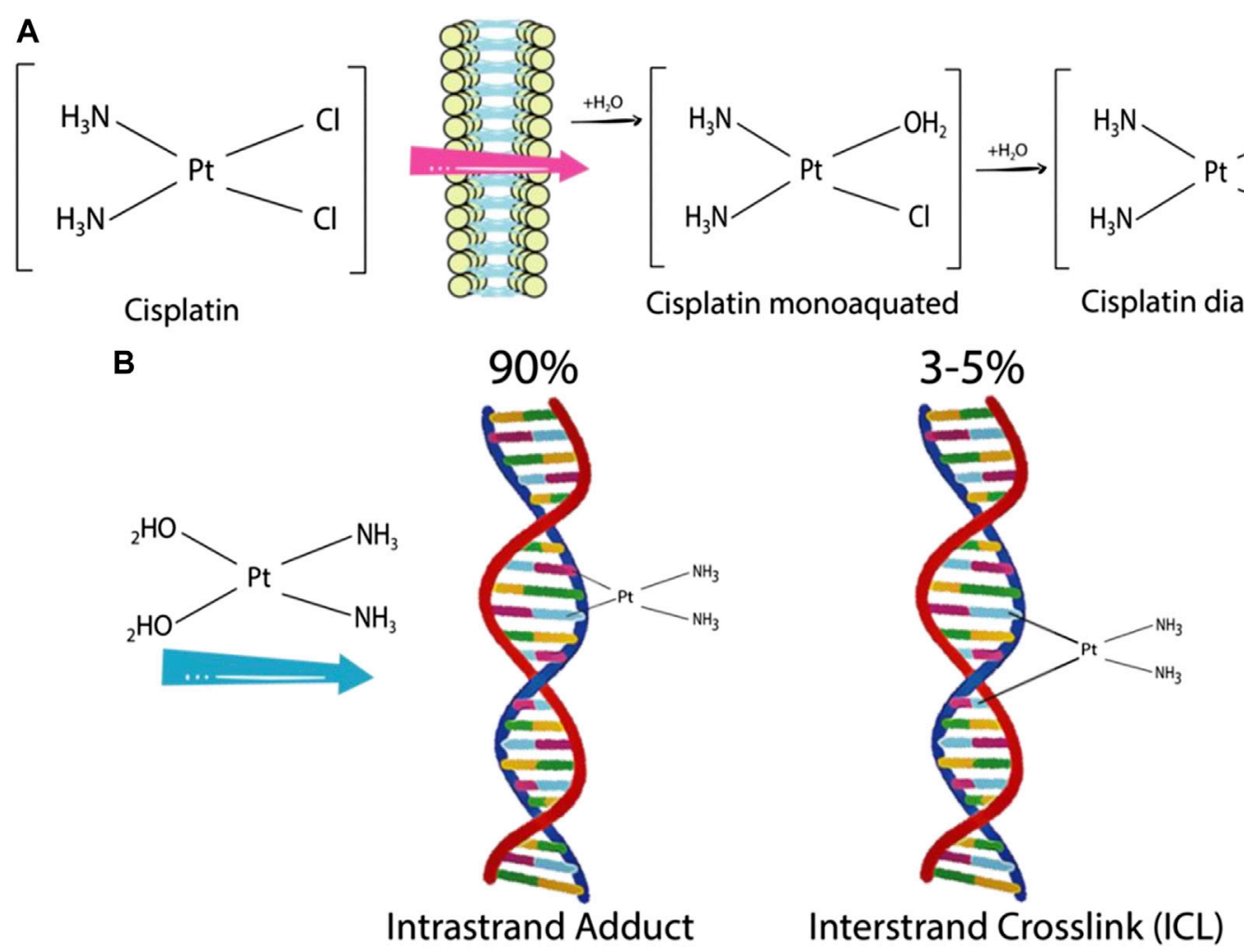

FIGURE 4 | Cisplatin activation and formation of cisplatin-DNA adducts (A) Cisplatin is mono- and diaquated upon entering a cell. (B) Cisplatin can form covalent bonds with DNA bases. An intra-strand adduct is formed when cisplatin cross links two bases of the same strand, whereas an inter-strand adduct is formed when cisplatin cross links two bases from different strands. The percentages indicate the frequency of each type of DNA lesion induced by cisplatin (Rocha et al., 2018).

chemoresistance and anti-apoptotic phenotype of tumor cells via delivery of exosomes.

Aside from exosomal enrichment of miRNAs conferring chemoresistance traits, miRNAs conferring chemosensitivity will be downregulated in resistant OSCC cells (Kulkarni et al., 2020). demonstrated that miR-30a was found to be significantly reduced in exosomes isolated from serum of OSCC patients, especially post cisplatin treatment. They also identified Beclin1 (autophagic marker) to be a binding target of miR-30a and are overexpressed in cisplatin-resistant OSCC cells. Exosomes from cisplatin-resistant cells that have been transfected with miR-30a mimics, when delivered to naïve cisplatin-resistant cells, caused a significant downregulation in Beclin1 and Bcl2 (antiapoptotic marker) expression, resulting in the sensitization of cisplatinresistant OSCC cells. Thus, this proves that downregulation of exosomal miR-30a is a chemoresistance trait in cisplatin-resistant OSCC cells.

These experiments provide extensive evidence on the role of exosomes in propagating anti-apoptotic signals, be it from neighboring OSCC cells or from the tumor microenvironment. Based on the above studies, the main exosomal content carrying apoptotic signals are found to be miRNAs. However, the role of other exosomal contents in mediating anti-apoptotic signals should also be investigated. This is important as CoutinhoCamillo et al. (2017) had demonstrated that the pathological and prognosis characteristics of OSCC cases cannot be predicted even with the expression profiling of the complete range of apoptosis-related proteins to date. It is possible that other exosomal contents may modulate apoptosis in mechanisms unknown to us.

Modulation of DNA Damage Repair (DDR) mechanisms. DNA damage repair (DDR) mechanisms play an essential role in anticancer drug resistance. Drugs such as cisplatin and carboplatin intercalate between DNA bases and introduce inter- and intra-strand linkages. These effects result in the inhibition of DNA synthesis, triggering DNA damage response and inducing apoptosis (Figure 4). The human DDR mechanism is regulated by various proteins responsible in recognition of DNA lesions, nucleotide excision and DNA ligation (Rocha et al., 2018). Exosomes play a role in modulating the DDR mechanism in cells under stress. Akiko et al. (2017) demonstrated that exosomes maintain cellular homeostasis by excreting harmful cytoplasmic DNA from cells (Akiko et al., 2017). Secretion of exosomes containing cytoplasmic DNA prevents aberrant activation of the DDR pathway, which is a precondition of apoptosis. The DDR response consists of checkpoint mechanisms that arrest cell cycle to allow the repair of damaged DNA, and if the severity is high, induce cell death via apoptosis. 
Cisplatin and many chemotherapeutic drugs are known for its ability to induce apoptosis via DDR (Rocha et al., 2018). Akiko et al. (2017) observed that exosomes released from human diploid fibroblasts (HDFs) have the potential to activate the DDR pathway in recipient pre-senescent HDFs depending on the amount of exosomes present (Akiko et al., 2017). Immunogold labeling of double-stranded DNA (dsDNA) along with transmission electron microscopy revealed the presence of DNA in the exosomes of multivesicular bodies (MVBs). Subsequent DNA sequencing analysis points toward the origin of these dsDNA fragments being chromosomal and not mitochondrial. Thus, it was concluded that the DNA fragments released via exosomes are harmful constituents in cells. Moreover, the amount of DNA in the exosomes was enriched when pre-senescent HDFs were treated with DNAdamaging agents such as doxorubicin (Akiko et al., 2017).

Interestingly, cisplatin-induced DNA lesions that stall replication forks can result in the formation of DNA doublestranded breaks (DSBs), resulting in chromosomal DNA fragments (Rocha et al., 2018). It can thus be hypothesized that the release of chromosomal DNA-containing exosomes is a mechanism reducing DDR activation which in the context of cancer cells, promotes cancer cell survival. It is possible that a similar mechanism as stated above is utilized by OSCC cells to reduce aberrant DDR mechanisms and reducing the cell's likelihood of undergoing apoptosis. However, similar studies on OSCC cell lines are needed to verify this. Mutschelknaus et al. (2016) discovered that tumor-derived exosomes were able to increase radioresistance in head and neck SCC (HNSCC) cells via increasing DSB repair (Mutschelknaus et al., 2016).

Although chemotherapy and radiotherapy are distinct from each other, both therapies induce DNA damage. Moreover, it has been demonstrated beforehand that exosomes from breast cancer cells can alter the phosphorylation status of DDR proteins (Dutta et al., 2014). Exosomes from irradiated and non-irradiated HNSCC BHY cells were transferred to irradiated BHY cells (Mutschelknaus et al., 2016). The number of DSB repair foci in the recipient BHY cells was analyzed and quantified after $6 \mathrm{~h}$ (Mutschelknaus et al., 2016). It was revealed that the number of repair foci was lower in the cells incubated with exosomes isolated from irradiated BHY cells, suggesting a quicker rate of DNA repair induced from these exosomes (Mutschelknaus et al., 2016). A similar phenomenon occurred in another HNSCC cell line $\mathrm{FaDu}$ (Mutschelknaus et al., 2016). Furthermore, exosomal release and uptake in HNSCC cells were shown to be higher under irradiation (Mutschelknaus et al., 2016), suggesting a putative mechanism involving exosomes which is triggered when HNSCC cells undergo DNA-damaging stress. It is important to note however, that (Mutschelknaus et al., 2016) did not investigate the exosomal content responsible in promoting the DSB repair.

DNA lesions resulting from damage induced by cisplatin and its analogues are repaired mainly via the nucleotide excision repair (NER) pathway (Rocha et al., 2018). However, a separate study conducted by Kothandapani et al. revealed that a different DDR pathway known as base excision repair (BER) is capable of mediating cisplatin cytotoxicity (Kothandapani et al., 2011).
Using ovarian carcinoma cells, breast cancer cells and HeLa cells, they demonstrated that BER maintains cytotoxicity of cisplatin by competing with the productive NER pathway (Kothandapani et al., 2011). One of the proteins facilitating the BER pathway is apurinic/apyrimidinic endonuclease 1 (APE1). APE1, a protein shown to be elevated in blood serum of OSCC patients (Yousafzai et al., 2018). Whether or not the protein in blood serum originates from exosomes remains unknown. However, Nath et al. (2017) demonstrated that APE1 is released from monocytic cells via exosomes (Nath et al., 2017). The presence of APE1 in isolated exosomes was examined by western blot analysis which showed that APE1 levels increased significantly in secreted exosomes compared to whole cell extract of the monocytic cells (Nath et al., 2017). Thus, it is possible that cell release of APE1 via exosomes is a chemoresistance mechanism inhibiting the BER pathway, allowing OSCC cells to respond to cisplatin-induced DNA damage via NER. However, this line of thought would need more experimental investigation based on OSCC models.

In order to prove the role of exosomes in modulating DDR mechanisms in chemoresistant OSCC, more studies establishing a direct link are needed. To date, there is only evidence showing the involvement of OSCC exosomes in mediating DNA repair, but none in a chemoresistance context.

\section{Immunomodulation by Exosomes}

Aside from modulating DDR mechanisms, exosomes have been shown to possess immunomodulatory effects (Momen-Heravi and Bala, 2018; Cai et al., 2019). Cellular crosstalk between tumor cells and their microenvironment which consists of immune cells such as monocytes and macrophages is important in tumor progression. Chemotherapeutic drugs such as cisplatin and doxorubicin inhibit DNA synthesis, which happens frequently in dividing cells. Thus, dividing immune cells often become targets of these drugs as well, weakening a patient's immune response against tumors. Moreover, tumor cells under chemotherapy-induced stress are capable of enhancing their immunosuppression abilities toward immune cells in the surrounding microenvironment. Baghdadi et al. demonstrated that Interleukin 34 (IL34) was produced by cancer cells under chemotherapy-induced stress to enhance local immunosuppression of tumor-associated macrophages (TAMs), increasing the ability of cancer cells to evade an immune response (Baghdadi et al., 2016).

A well investigated pathway which mediates the critical changes characteristic of innate and adaptive immune responses is the nuclear factor kappa-light-chain-enhancer of activated B cells (NF- $\kappa$ B) pathway (Hayden et al., 2006). NF- $\kappa B$ is a family composed of five related transcription factors: $\mathrm{p} 50$, p52, RelA, c-Rel and RelB (Gilmore, 2006). These transcription factors possess a $\mathrm{N}$-terminal DNA-binding domain that binds to target DNA sequences called $\kappa \mathrm{B}$ sites to regulate gene expression (Jiang et al., 2009). In a study conducted by Momen-Heravi and Bala (2018), exosomes from OSCC cells can reprogram monocytes via the NF-кB pathway (Momen-Heravi and Bala, 2018). They discovered that exosomes released from CAL27 cells both in normal and under ethanol conditions contained a high signal of 
miR-21 (Momen-Heravi and Bala, 2018). Exosomes released from THP1 monocytes however, does not contain a similarly high level of miR-21, implying a specific sorting mechanism involved in packaging of miR-21 into exosomes in OSCC cells (Momen-Heravi and Bala, 2018). Fluorescence-labelled exosomes from CAL27 cells were observed to be taken up by THP1 monocytes (Momen-Heravi and Bala, 2018). This was further proven by the observation that Cytochalasin D (an inhibitor of endocytic pathways and exosome uptake) treatment resulted in decrease of miR-21 level in THP1 monocytes (Momen-Heravi and Bala, 2018). Electrophoretic mobility shift assay (EMSA) of specific NF- $\kappa \mathrm{B}$ consensus sequences showed activation of NF- $\kappa \mathrm{B}$ p50 heterodimers in THP1 monocytes (Momen-Heravi and Bala, 2018). It was postulated that exosomes released from CAL27 were capable of reprogramming the monocytes. Monocyte chemoattractant protein-1 (MCP1) which molds the tumor microenvironment and promotes tumorigenesis (Yoshimura, 2017) was shown to increase significantly in recipient THP1 monocytes after co-culture of exosomes derived from CAL27 (Momen-Heravi and Bala, 2018). The level of other prooncogenic factors such as matrix metallopeptidase 9 (MMP9), cyclooxygenase-2 (COX2) mRNA levels, vascular endothelial growth factor (VEGF) and interleukin-6 (IL6) were increased after co-culture with exosomes (Momen-Heravi and Bala, 2018). However, preliminary size characterization of the EVs released from CAL27 cells via nanoparticle tracking analysis revealed that there is a mix of both exosomes and microvesicles. The inference that THP1 monocytes are reprogrammed by the microvesicles carrying miR-21 is also viable. Notwithstandingl, these observations still support the conclusion that exosomes from OSCC cells are capable of modulating the immune function of surrounding monocytes and establishing a pro-inflammatory milieu via miR-21 (Momen-Heravi and Bala, 2018). Chronic inflammation promotes tumorigenesis by disabling the immune system from attacking tumor cells, inducing cell proliferation and genetic instability leading to oncogenic mutations (Xia et al., 2014).

Aside from monocytes, exosomes from OSCC can also reprogram macrophages. Cai et al. (2019) demonstrated that OSCC-derived exosomes containing miR-29a-3p promote M2 subtype macrophage polarization (Cai et al., 2019). Exosomes isolated from SCC9 and CAL27 OSCC cells were co-cultured with macrophages (Cai et al., 2019). The expression level of M2 subtype macrophage marker proteins such as CD163, CD206, Arg-1 and IL-10 increased after co-culturing (Cai et al., 2019). After that, the conditioned-medium of co-culturing exosomes and macrophages was used to culture SCC9 and CAL27 cells (Cai et al., 2019). Transwell assay conducted on SCC9 and CAL27 showed an increase in cell invasion and proliferation, proving that the M2 subtype macrophage polarization promotes tumorigenesis and pro-metastatic environment for OSCC cells (Cai et al., 2019). These results were also supported by Kazumasa et al. (Mori et al., 2011). They performed immunohistochemical analysis to identify M2 TAMs in surgically resected OSCC specimens from 50 patients and discovered a positive correlation between the proportion of M2 TAMs and the pathological grade of the OSCC specimen (Mori et al., 2011).
Thus, it can be concluded that immunomodulation mediated by exosomes is an important topic of investigation and should be included in the search for therapeutic strategies against OSCC chemoresistance.

\section{Epithelial-Mesenchymal Transition (EMT)}

The epithelial-mesenchymal transition (EMT) is a conserved process marked by the loss of epithelial characteristics and gain of mesenchymal phenotype of a cell at a genetic, epigenetic and morphological level (Greening et al., 2015). It is characterized by the downregulation of cell adhesion molecules E-cadherin and $\beta$-catenin with the concomitant upregulation of mesenchymal markers $\mathrm{N}$-cadherin and vimentin (Thomas et al., 2018). EMT was initially thought to be confined within embryological development before its conception as a mechanism endowing metastatic and invasive properties to a tumor (Greening et al., 2015). During EMT, the epithelial cells lose apical-basal polarity and epithelial cell-cell contacts such as tight junctions, adherens junctions and the actin cytoskeletal architecture are disassembled and reorganized (Lu and Kang, 2019). The resulting mesenchymal cells attain a spindle-shape morphology and gain motility. The cellular changes are accompanied by a change in the expression of epithelial genes. For instance, cells undergoing EMT will express matrix metalloproteinases (MMPs) to degrade and invade the basal extracellular matrix (Lu and Kang, 2019). Figure 5 below briefly outlines the main steps of the EMT process.

There is increasing evidence that suggests EMT contributes to chemoresistance. Earlier studies have established the connection between transcription factors regulating expression of EMT genes and drug sensitivity (Vega et al., 2004; Arumugam et al., 2009; Kurrey et al., 2009). The studies demonstrated an increase in drug sensitivity when the transcription factors are repressed. Recent studies utilizing murine models of pancreatic and breast cancers demonstrate that EMT is dispensable for metastasis to occur, but contributes strongly to chemoresistance (Xiaofeng et al., 2015; Shields, 2018). Clinicopathological studies and immunohistochemical analysis of OSCC EMT markers have also supported the idea that EMT is not a necessary step for metastasis, but may be responsible for other properties such as chemoresistance and the formation of stem cell-like properties (Tarin et al., 2005; Chui, 2013; Zidar et al., 2018).

EMT can be mediated by exosomes in OSCC cells undergoing hypoxic stress. Hypoxia has often been postulated to act as an environmental pressure resulting in malignant evolution of tumor cells (Lu and Kang, 2010). The metastatic process of tumors under hypoxia was accelerated via EMT (Lu and Kang, 2010; Li et al., 2016). Li et al. demonstrated that hypoxic OSCC cells deliver miR-21 via exosomes to induce EMT in normoxic cells (Li et al., 2016). Immunoblotting for CD63 revealed that hypoxia induced exosome release from CAL27 and SCC9 OSCC cell lines ( $\mathrm{Li}$ et al., 2016). These exosomes were isolated and incubated along with normoxic cells. The migration and invasion of the cells measured using wound healing assays increased ( $\mathrm{Li}$ et al., 2016). This along with the increased expression of EMT marker vimentin proves that exosomes from hypoxic OSCC cells can induce EMT in recipient normoxic cells (Li et al., 2016). 


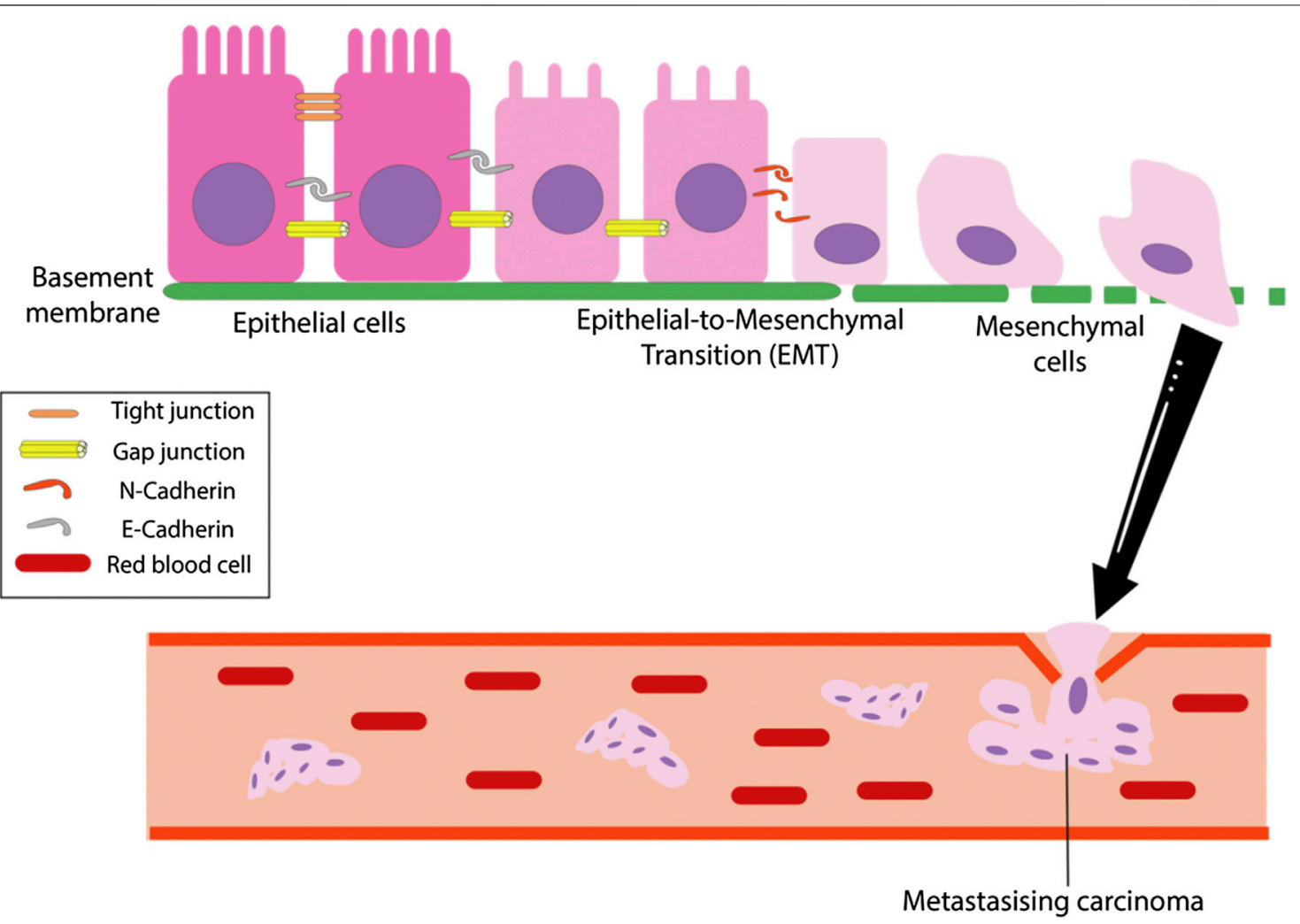

FIGURE 5 | The overall EMT process. The epithelial-mesenchymal transition (EMT) is the process which tumor cells change from epithelioid to mesenchymal cell morphology and is marked by a loss of cell polarity, tight, gap and adherent junctions in epithelial cells, resulting in mesenchymal cells that has increased migratory and invasive capabilities (Li et al., 2019).

MiRNA expression profile and RT-PCR revealed that miR-21 was expressed higher in hypoxic exosomes compared to normoxic exosomes (Li et al., 2016). Hence, Li et al. (2016) concluded that miR-21 was the main mediator inducing EMT changes in normoxic cells from hypoxic cells (Li et al., 2016). This theory was further validated by murine xenograft models, where injection of hypoxic exosomes into the xenograft tumors increased the growth, weight of tumor and caused an overexpression of miR-21 compared to normoxic exosomes ( $\mathrm{Li}$ et al., 2016).

A recent study conducted by (Kirave et al., 2020) further elucidated the EMT modulatory properties of OSCC exosomes. In this study, it was shown that exosomes from cisplatin-resistant OSCC cells carrying miR-155 are capable of inducing miR-155 overexpression in sensitive OSCC cells. The binding target of miR-155 was verified via bioinformatics and transfection experiments to be $\mathrm{FOXO} 3$, thereby inhibition of this protein leads to upregulation of EMT markers. Furthermore, exosomes derived from cisplatin-resistant cells transfected with miR-155 mimics are shown to be enriched in miR-155. When sensitive OSCC cells are treated with these exosomes, protein and mRNA levels of FOXO3 decreased and cisplatin resistance of the cells were enhanced. These results proved a close link between EMT and chemoresistance, and the important role played by exosomes in facilitating EMT in OSCC.
Besides exosomal miR-21 and miR-155, other EMTmodulating factors which may be potential cargoes of OSCC exosomes are epidermal growth factor (EGF), CD47, miR-222 and other EMT transcription factors (EMT-TFs) (Nieto et al., 2016; Ouyang et al., 2017; Fujiwara et al., 2018; Pai et al., 2019). A separate study done by Chang et al. showed that the miRNA let7d, a known tumor suppressor was repressed as EMT-promoting transcription factors Twist and Snail are overexpressed in OSCC (Chang et al., 2011). Cell viability experiments showed that ectopic overexpression of let-7d suppressed the chemoresistance of OSCC cells to cisplatin and paclitaxel (Chang et al., 2011). EMT-TFs are known to be exosomal cargoes (Nieto et al., 2016). Thus, it is possible that EMT-TFs can be transported as exosomal cargoes to distant cells and promote EMT and chemoresistance phenotypes. However, future experiments are needed to establish the presence of these EMT-TFs in OSCC exosomes and investigate their effects on recipient cells.

\section{Exosomes From Cancer Stem Cells}

The cancer stem cell (CSC) hypothesis postulates that CSCs are responsible for the maintenance and recurrence of tumors (Yoo and Kwon, 2015). This small subset of cancer cells possess properties such as self-renewal, slow replication, intrinsic resistance to chemotherapy and an ability to give rise to 
differentiated progeny (O'flaherty et al., 2012). Cellular dormancy allows CSCs to escape antineoplastic treatment as they are recruited to the anti-mitotic and quiescent G0-phase (Kleffel and Schatton, 2013). A lower proliferation rate would reduce the efficacy of DNA-damaging chemotherapeutic agents. Several studies of other cancer models have shown that exosomes are capable of conferring stemness to cancer cells (Koch et al., 2014; Bliss et al., 2016). Mesenchymal stem cells (MSCs)-derived exosomes containing miR-222 induced quiescence in breast cancer cells in vitro and in vivo, thus conferring drug resistance (Bliss et al., 2016). In an in vitro model of diffuse large B-cell lymphoma (DLBCL), exosomes containing Wingless-related integration site (Wnt) signaling factor from DLBCL can induce a CSC phenotype in recipient cells (Koch et al., 2014). CSCs are able to endure environmental stress such as radiation and hypoxia by maintaining their stemness via exosomes containing Hedgehog, Wnt, $\beta$-catenin and other mRNAs and proteins (Fatima and Nawaz, 2015). These exosomal cargoes can sustain the self-renewal and clonogenic properties of CSCs.

CSC-like phenotype can be induced by microRNA such as miR-146a (Roychoudhury et al., 2018). Roychoudhury et al. demonstrated that miR-146a overexpression enhances the stemness of OSCC cells by augmenting CD44 and CD24 levels (Roychoudhury et al., 2018). CD24 was identified as a functional target of miR-146a (Roychoudhury et al., 2018). Roychoudhury et al. (2018) demonstrated that miR-146a confers stemness via suppression of CD24. Mechanistic analysis revealed that higher CD24 levels inhibit AKT phosphorylation leading to degradation of $\beta$-catenin, an important factor for cancer stemness (Roychoudhury et al., 2018). However, studies to verify whether exosomes isolated from OSCC or the surrounding population of CSCs contain miR-146a are needed. Whether or not exosomes play a role in conferring cancer stemness in OSCC remains an unsolved mystery.

\section{Microvesicles}

Microvesicles are heterogenous, membranous sacs that are shed directly from the plasma membrane of a cell (Raposo and Stoorvogel, 2013). They are a distinct group of extracellular vesicles that are much larger in size (100-1,000 nm) compared to exosomes. Like exosomes, they also carry various forms of cargo including proteins, miRNA and RNA transcripts.

The biogenesis of microvesicles involves the outward blebbing and pinching of the plasma membrane. The process is accompanied by localized changes in the plasma membrane protein and lipid components which are involved in modulating membrane curvature (McMahon and Boucrot, 2015). Synergistic action of multiple mechanisms such as asymmetric partitioning of transmembrane domains, protein scaffolding and cytoskeletal reorganization both at the nanoscopic and macroscopic level mediate microvesicle budding (Harvey and Jennifer, 2005). Due to the heterogeneity of their size, microvesicles can be generated by various distinct mechanisms that may partially overlap with those of exosome biogenesis. For example, sphingomyelinases that convert sphingomyelin to ceramide are shown to be both responsible in MVB formation and the budding of microvesicles (Trajkovic et al., 2008; Bianco et al., 2009).

While it has not been extensively studied, microvesicles have been shown to modulate drug resistance in cancer cells, making them another factor to consider in combating drug resistance. Microvesicles isolated from OSCC have been shown to be larger and enriched than normal control cells. Their cargo contains oncogenic miRNA such as miR-21, miR-27a, miR-27b, and miR155 (Momen-Heravi and Bala, 2018). One of them, miR-21, has been strongly evidenced to contribute toward OSCC chemoresistance via the STAT3/miR-21 axis (Xuan et al., 2014). Besides that, the miRNA signatures have been evidenced to induce pro-inflammatory phenotype in the tumor microenvironment by reprogramming monocytes via the NF- $\kappa B$ pathway (Momen-Heravi and Bala, 2018).

\section{Microvesicle-Mediated Chemoresistance}

The involvement of microvesicles (MVs) in chemoresistance properties are not as extensive as exosomes. However, they have been implicated in intercellular communication within the tumor microenvironment such as stromal and immune cells, transport of drug efflux proteins, drug sequestration and anti-apoptotic signaling (Kim et al., 2005; Abid Hussein et al., 2007; Gong et al., 2013; Zhang et al., 2014a; Zhang et al., 2014b). The pathway involved for the microvesicle-mediated chemoresistance is through the cargoes that is packed into MVs. Transmembrane, soluble proteins, lipids or nucleic acids that are packed into MVs shedding from plasma membrane could be endocytosed by recipient cells and results in the delivery of its cargoes into the recipient cells, thus transferring the information and influence the cancer development (Bian et al., 2019).

MVs isolated from A2780 ovarian cancer cells were shown to carry P-glycoprotein (P-gp), a protein overexpressed in multidrug resistance (MDR) phenotype (Zhang et al., 2014a). Intercellular transfer of MVs mediate the 'sharing' of P-gp which confers paclitaxel-resistance to recipient A2780 cells. Aside from that, MVs have also been shown to sequester drugs and reduce the cytosolic free drug concentration in breast adenocarcinoma and acute lymphoblastic leukemia cells (Gong et al., 2013). MVs have also shown to carry anti-apoptotic potential. A study conducted by Abid Hussein et al. demonstrated that inhibiting the release of caspase-3-containing MVs triggers endothelial cell detachment and subsequent apoptosis, thus showing that release of caspase-3containing MVs is an important anti-apoptotic mechanism (Abid Hussein et al., 2007). In addition, MV-carrying EGFR can be taken up by endothelial cells which in turn activates MAPK and Akt pathways, this will lead to the endogenous expression of VEGF, followed by the activation of VEGF receptor-2 and tumor angiogenesis (Al-Nedawi et al., 2009).

Whether or not MVs of OSCC carry out similar functions is yet to be investigated. On the other hand, MVs from OSCC have already been proven to induce apoptosis of T lymphocytes (Kim et al., 2005). OSCC patients often have a high proportion of $\mathrm{T}$ lymphocytes undergoing apoptosis (Kim et al., 2005). T cells found at tumor sites often experience dysregulation of signaling factors such as TCR- $\zeta$ downregulation (Reichert et al., 2002). Kim et al. (2005) demonstrated that Fas Ligand (FasL)-Positive MVs isolated 
from the sera of OSCC patients can induce apoptosis of $\mathrm{T}$ lymphocytes, enabling the OSCC cells with immunosuppression capabilities (Kim et al., 2005). Aside from that, Momen-Heravi and Bala (2018) demonstrated that MVs from CAL27 cells carrying miR-21 are capable of reprogramming monocytes via the NF- $\kappa B$ pathway (Momen-Heravi and Bala, 2018). However as stated above, the EVs isolated from Momen-Heravi et al. contain a mixture of exosomes and MVs (Momen-Heravi and Bala, 2018).

The involvement of MVs in facilitating intercellular communication, changes in tumor microenvironment and drug resistance in OSCC needs more investigation. Besides that, improvements and optimisation of isolation protocols and methodologies are of paramount importance. There is a need to achieve an EV subtype yield with the highest purity and integrity in order to avoid studying MVs and exosomes in the same context. Characterization methods of EVs need continual improvement in order to distinguish between MVs and exosomes. The distinguishing of MVs and exosomes from each other will avoid wrongly attributing EV cargoes and signaling pathways to an EV subtype.

\section{CONCLUSION}

The involvement of EVs in mediating chemoresistance in OSCC still requires ample investigation. To date, only a handful of direct links implicating EV involvement in OSCC chemoresistance have been established. Nevertheless, these findings provide sufficient basis that both exosomes and microvesicles play an important role in several chemoresistance mechanisms. In this review, we have discussed several roles of $\mathrm{EVs}$ in the regulation of chemoresistance in OSCC including its exosomal content, drug efflux by EVs, alteration of vesicular $\mathrm{pH}$, anti-apoptotic signaling transmitted by EVs, modulation of DDR mechanisms, immunomodulation by exosomes, transport of EMT promoting genes and lastly, microvesicle-mediated chemoresistance.

In summation of the above findings related to exosomes, exosomal content such as miR-21 and miR-24 are recognized as key oncogenic factors mediating OSCC cell survival. Aside from that, drug efflux by EVs for instance cisplatin efflux mediated by copper transporters ATP7A and ATP7B remains an active field of investigation. Novel studies suggest that cisplatin may be transported out by ATP7B via lysosomal exocytosis, albeit similar studies done on OSCC are yet to be made. Alteration of vesicular $\mathrm{pH}$ by vacuolar ATPases residing on the endomembrane presents a viable mechanism of OSCC chemoresistance as cisplatin sensitivity depends on the acidity of intracellular environment.

Besides that, exosomes from cancer-associated fibroblasts can deliver anti-apoptotic signaling miRNAs that alter expression of genes regulating apoptosis. In addition, exosomal release of chromosomal DNA may be a potential mechanism utilized by OSCC to reduce aberrant DDR activation and apoptosis. Furthermore, exosomes from OSCC have been shown to be capable in reprogramming monocytes via the NF- $\kappa B$ pathway and macrophages via miR-29a-3p, thus mediating immunosuppression of the tumor microenvironment. Aside from that, an emerging perspective suggests that epithelial-tomesenchymal transition is a chemoresistant mechanism in addition to being a metastatic process. Exosomes from OSCC cells carrying EMT-promoting cargoes such as miR-21, miR-155 (experimentally proven to confer chemoresistance) and EMTtranscription factors may confer chemoresistance phenotypes to recipient cells. Last but not least, exosomal miR-146a are implicated in enhancing stemness in OSCC cells. Cancer stem cells are known for self-renewal and low proliferation properties, thus making them harder targets for DNA-damaging drugs. Findings in relation to microvesicle-mediated chemoresistance in OSCC however, are limited. Nevertheless, notable findings of MV-mediated chemoresistance include apoptosis induction of $\mathrm{T}$ lymphocytes and reprogramming of monocytes by OSCC MVs. However, it is important that EV characterization methods be standardized across laboratories to distinguish MVs from exosomes.

In addition to that, the various pathways of biogenesis and endocytic mechanisms of EVs need to be mapped in order for EV inhibition to be considered as a synergistic complement to chemotherapy. Current research has no guarantee that EV inhibition will fully cripple tumor progression. In addition, due to the heterogeneity of OSCC cases, there is no guarantee that a study done on a few OSCC cell lines are fully applicable to all OSCC cases. Current molecular diagnostics utilizing tumor biomarkers are unable to accurately predict tumor evolution and behavior due to intra- and inter-tumor heterogeneity (Ogino et al., 2012). The complex interactions between tumor cells and the host cells, which is influenced by genomic variation and environmental factors such as diet and lifestyle presents a longstanding impasse in clinical diagnostics and treatment strategies. With that in mind, it is of paramount importance that deeper studies probing into the mechanisms of EV-mediated chemoresistance are carried out to improve the resolution of molecular characterizations on OSCC cases.

\section{AUTHOR CONTRIBUTIONS}

Z-JL and X-HK performed searching, analyzed data, and wrote the manuscript. HB, BG and W-LL contributed to the conceptual idea, reviewed the drafts, supervised the writing process, and provided important information for the completion of this manuscript. LM and P-TL reviewed the drafts and refined the manuscript. All the authors revised and approved the final version of the manuscript.

\section{FUNDING}

This work was supported by Fundamental Research Grant Scheme Grants (FRGS/1/2019/SKK08/MUSM/02/4) from the Ministry of Higher Education Malaysia.

\section{ACKNOWLEDGMENTS}

Special thanks to Prof Ian Paterson from University of Malaya, Malaysia for his generous help in English editing of the article. 


\section{REFERENCES}

Abid Hussein, M. N., Böing, A. N., Sturk, A., Hau, C. M., and Nieuwland, R. (2007). Inhibition of microparticle release triggers endothelial cell apoptosis and detachment. Thromb. Haemost. 98 (5), 1096. doi:10.1160/TH05-04-0231

Ahmed, K. A., and Xiang, J. (2011). Mechanisms of cellular communication through intercellular protein transfer. J. Cell Mol. Med. 15 (7), 1458-1473. doi:10.1111/j.1582-4934.2010.01008.x

Akiko, T., Ryo, O., Koji, N., Yuka, K., Aki, H., Shin, Y., et al. (2017). Exosomes maintain cellular homeostasis by excreting harmful DNA from cells. Nature Commun. 8 (7), 121-129. doi:10.1038/ncomms15287

Al-Nedawi, K., Meehan, B., Kerbel, R. S., Allison, A. C., and Rak, J. (2009). Endothelial expression of autocrine VEGF upon the uptake of tumor-derived microvesicles containing oncogenic EGFR. Proc. Natl. Acad. Sci. U. S. A. 106 (10), 3794-3799. doi:10.1073/pnas.0804543106

Alnuaimi, A. D., Wiesenfeld, D., O’brien-Simpson, N. M., Reynolds, E. C., and McCullough, M. J. (2015). Oral Candida colonization in oral cancer patients and its relationship with traditional risk factors of oral cancer: a matched casecontrol study. Oral Oncol. 51 (2), 139-145. doi:10.1016/j.oraloncology.2014. 11.008

Arumugam, T., Ramachandran, V., Fournier, K. F., Wang, H., Marquis, L., Abbruzzese, J. L., et al. (2009). Epithelial to mesenchymal transition contributes to drug resistance in pancreatic cancer. Cancer Res. 69 (14), 5820. doi:10.1158/0008-5472.CAN-08-2819

Baghdadi, M., Wada, H., Nakanishi, S., Abe, H., Han, N., Putra, W. E., et al. (2016). Chemotherapy-induced IL34 enhances immunosuppression by tumorassociated macrophages and mediates survival of chemoresistant lung cancer cells. Cancer Res. 76 (20), 6030. doi:10.1158/0008-5472.CAN-16-1170

Baixauli, F., Lopez-Otin, C., and Mittelbrunn, M. (2014). Coordinated mechanisms for the maintenance of cellular fitness. Front. Immunol. 5, 133. doi:10.3389/ fimmu.2014.00403

Bartel, D. P. (2004). MicroRNAs: genomics, biogenesis, mechanism, and function. Cell 116 (2), 281-297. doi:10.1016/S0092-8674(04)00045-5

Becelli, R., Renzi, G., Morello, R., and Altieri, F. (2007). Intracellular and extracellular tumor $\mathrm{pH}$ measurement in a series of patients with oral cancer. J. Craniofac. Surg. 18 (5), 1051-1054. doi:10.1097/scs.0b013e3180de63eb

Belcher, R., Hayes, K., Fedewa, S., and Chen, A. Y. (2014). Current treatment of head and neck squamous cell cancer. J. Surg. Oncol. 110, 551-574. doi:10.1002/ jso. 23724

Beretta, G. L., Gatti, L., Tinelli, S., Corna, E., Colangelo, D., Zunino, F., et al. (2004). Cellular pharmacology of cisplatin in relation to the expression of human copper transporter CTR1 in different pairs of cisplatin-sensitive and -resistant cells. Biochem. Pharmacol. 68 (2), 283-291. doi:10.1016/j.bcp.2004.03.022

Bian, X., Xiao, Y.-T., Wu, T., Yao, M., Du, L., Ren, S., et al. (2019). Microvesicles and chemokines in tumor microenvironment: mediators of intercellular communications in tumor progression. Mol. Cancer 18 (1), 50. doi:10.1186/ s12943-019-0973-7

Bianco, F., Perrotta, C., Novellino, L., Francolini, M., Riganti, L., Menna, E., et al. (2009). Acid sphingomyelinase activity triggers microparticle release from glial cells. EMBO J. 28 (8), 1043-1054. doi:10.1038/emboj.2009.45

Bliss, S. A., Sinha, G., Sandiford, O. A., Williams, L. M., Engelberth, D. J., Guiro, K., et al. (2016). Mesenchymal stem cell-derived exosomes stimulate cycling quiescence and early breast cancer dormancy in bone marrow. Cancer Res. 76 (19), 5832. doi:10.1158/0008-5472.CAN-16-1092

Böing, A. N., Stap, J., Hau, C. M., Afink, G. B., Ris-Stalpers, C., Reits, E. A., et al. (2013). Active caspase- 3 is removed from cells by release of caspase-3-enriched vesicles. BBA Mol. Cell Res. 1833 (8), 1844-1852. doi:10.1016/j.bbamcr.2013. 03.013

Boudreau, N., Werb, Z., and Bissell, M. (1996). Suppression of apoptosis by basement membrane requires three-dimensional tissue organization and withdrawal from the cell cycle. Proc. Natl. Acad. Sci. U. S. A 93 (8), 3509. doi:10.1073/pnas.93.8.3509

Bunnell, A., Pettit, N., Reddout, N., Sharma, K., O’Malley, S., Chino, M., et al. (2010). Analysis of primary risk factors for oral cancer from select US states with increasing rates. Tob. Induc. Dis. 8 (1), 5. doi:10.1186/1617-9625-8-5

Cai, J., Qiao, B., Gao, N., Lin, N., and He, W. (2019). Oral squamous cell carcinoma-derived exosomes promote M2 subtype macrophage polarization mediated by exosome-enclosed miR-29a-3p. Am. J. Physiol. 316 (5), C731. doi:10.1152/ajpcell.00366.2018

Camakaris, J., Voskoboinik, I., and Mercer, J. F. (1999). Molecular mechanisms of copper homeostasis. Biochem. Biophys. Res. Commun. 261 (2), 225-232. doi:10. 1006/bbrc.1999.1073

Carvalho, A. L., Kowalski, L. P., Agra, I. M. G., Pontes, E., Campos, O. D., and Pellizzon, A. C. A. (2005). Treatment results on advanced neck metastasis (N3) from head and neck squamous carcinoma. Otolaryngol. Head Neck Surg. (Tokyo) 132 (6), 862-868. doi:10.1016/j.otohns.2005.01.034

Chang, C. J., Hsu, C. C., Chang, C. H., Tsai, L. L., Chang, Y. C., Lu, S. W., et al. (2011). Let-7d functions as novel regulator of epithelial-mesenchymal transition and chemoresistant property in oral cancer. Oncol. Rep. 26 (4), 1003-1010. doi:10.3892/or.2011.1360

Chang, S. S., Jiang, W. W., Smith, I., Poeta, L. M., Begum, S., Glazer, C., et al. (2008). MicroRNA alterations in head and neck squamous cell carcinoma. Int. J. Cancer 123 (12), 2791-2797. doi:10.1002/ijc.23831

Chen, D., Yan, W., Liu, Z., Zhang, Z., Zhu, L., Liu, W., et al. (2016). Downregulation of miR-221 enhances the sensitivity of human oral squamous cell carcinoma cells to Adriamycin through upregulation of TIMP3 expression. Biomed. Pharmacother. 77, 72-78. doi:10.1016/j.biopha. 2015.12.002

Chui, M. H. (2013). Insights into cancer metastasis from a clinicopathologic perspective: epithelial-Mesenchymal Transition is not a necessary step. (Hoboken). Int. J. Cancer 132, 1487-1495. doi:10.1002/ijc.27745

Chung, C. H., Parker, J. S., Karaca, G., Wu, J., Funkhouser, W. K., Moore, D., et al. (2004). Molecular classification of head and neck squamous cell carcinomas using patterns of gene expression. Cancer Cell 5 (5), 489-500. doi:10.1016/ S1535-6108(04)00112-6

Colombo, M., Raposo, G., and Thry, C. (2014). Biogenesis, secretion, and intercellular interactions of exosomes and other extracellular vesicles. Annu. Rev. Cell Dev. Biol. 30 (1), 255-289. doi:10.1146/annurev-cellbio-101512-122326

Coutinho-Camillo, C. M., Lourenco, S. V., Puga, R. D., Damascena, A. S., Teshima, T. H. N., Kowalski, L. P., et al. (2017). Profile of apoptotic proteins in oral squamous cell carcinoma: a cluster analysis of 171 cases. Applied Cancer Research 37 (1), 33. doi:10.1186/s41241-016-0008-2

Cusan, M., Mungo, G., De Marco Zompit, M., Segatto, I., Belletti, B., and Baldassarre, G. (2018). Landscape of CDKN1B mutations in luminal breast cancer and other hormone-driven human tumors. Front. Endocrinol. 9, 393. doi:10.3389/fendo.2018.00393

D'Cruz, A. K., Vaish, R., and Dhar, H. (2018). Oral cancers: current status. Oral Oncol. 87, 64-69. doi:10.1016/j.oraloncology.2018.10.013

Da Silva, S. D., Hier, M., Mlynarek, A., Kowalski, L. P., and Alaoui-Jamali, M. A. (2012). Current and emerging therapeutic approaches. Front. Pharmacol. 3, 149. doi:10.3389/fphar.2012.00149

Didonato, M., and Sarkar, B. (1997). Copper transport and its alterations in Menkes and Wilson diseases. Biochim. Biophys. Acta (BBA) Mol. Basis Dis. 1360 (1), 3-16. doi:10.1016/S0925-4439(96)00064-6

Dillon, L. M., and Miller, T. W. (2014). Therapeutic targeting of cancers with loss of PTEN function. Curr. Drug Targets 15 (1), 65. doi:10.2174/ 1389450114666140106100909

Dutta, S., Bandyopadhyay, C., Dutta, D., and Chandran, B. (2014). Interactions between exosomes from breast cancer cells and primary mammary epithelial cells leads to generation of reactive oxygen species which induce DNA damage response, stabilization of p53 and autophagy in epithelial cells. PloS One 9 (5), e97580. doi:10.1371/journal.pone.0097580

Dvořáková, M., Karafiát, V., Pajer, P., Kluzáková, E., Jarkovská, K., Peková, S., et al. (2013). DNA released by leukemic cells contributes to the disruption of the bone marrow microenvironment. Oncogene 32 (44), 121. doi:10.1038/onc.2012.553

Elaiwy, O., El Ansari, W., AlKhalil, M., and Ammar, A. (2020). Epidemiology and pathology of oral squamous cell carcinoma in a multi-ethnic population: retrospective study of 154 cases over 7 years in Qatar. Ann. Med. Surg. (Lond.) 60, 195-200. doi:10.1016/j.amsu.2020.10.029

Fatima, F., and Nawaz, M. (2015). Stem cell-derived exosomes: roles in stromal remodeling, tumor progression, and cancer immunotherapy. Cancer Commun. 34 (12), 1-13. doi:10.1186/s40880-015-0051-5

Friedman, R. (2016). Drug resistance in cancer: molecular evolution and compensatory proliferation. Oncotarget 7 (11), 11746. doi:10.18632/ oncotarget.7459 
Friedrich, R. E., Punke, C., and Reymann, A. (2004). Expression of multi-drug resistance genes (mdrl, mrpl, bcrp) in primary oral squamous cell carcinoma. In Vivo 18 (2), 133-147. doi:10.1532/ijh97.e0624

Fujiwara, T., Eguchi, T., Sogawa, C., Ono, K., Murakami, J., Ibaragi, S., et al. (2018). Carcinogenic epithelial-mesenchymal transition initiated by oral cancer exosomes is inhibited by anti-EGFR antibody cetuximab. Oral Oncol. 86, 251-257. doi:10.1016/j.oraloncology.2018.09.030

Furness, S., Glenny, A.-M., Worthington, H. V., Pavitt, S., Oliver, R., Clarkson, J. E., et al. (2011). Interventions for the treatment of oral cavity and oropharyngeal cancer: chemotherapy. Cochrane Database Syst. Rev. 14, 12-19. doi:10.1002/ 14651858.CD006386.pub3

Gajos-Michniewicz, A., Duechler, M., and Czyz, M. (2014). MiRNA in melanomaderived exosomes. Cancer Lett. 347 (1), 29-37. doi:10.1016/j.canlet.2014.02.004

Gao, W., and Han, J. (2018). Overexpression of ING5 inhibits HGF-induced proliferation, invasion and EMT in thyroid cancer cells via regulation of the c-Met/PI3K/Akt signaling pathway. Biomed. Pharmacother. 98, 265-270. doi:10.1016/j.biopha.2017.12.045

Gaudelot, K., Gibier, J.-B., Pottier, N., Hémon, B., Van Seuningen, I., Glowacki, F., et al. (2017). Targeting miR-21 decreases expression of multi-drug resistant genes and promotes chemosensitivity of renal carcinoma. Tumor Biology 39 (7), 133-139. doi:10.1177/1010428317707372

Gharat, S. A., Momin, M., and Bhavsar, C. (2016). Oral squamous cell carcinoma: current treatment strategies and nanotechnology-based approaches for prevention and therapy. Crit. Rev. Ther. Drug Carrier Syst. 33 (4), 363-400. doi:10.1615/CritRevTherDrugCarrierSyst

Gilmore, T. D. (2006). Introduction to NF-kB: players, pathways, perspectives. Oncogene 25 (51), 6680. doi:10.1038/sj.onc.1209954

Gong, J., Luk, F., Jaiswal, R., George, A. M., Grau, G. E. R., and Bebawy, M. (2013). Microparticle drug sequestration provides a parallel pathway in the acquisition of cancer drug resistance. Eur. J. Pharmacol. 721 (1-), 116-125. doi:10.1016/j. ejphar.2013.09.044

Gould, S. J., Booth, A. M., and Hildreth, J. E. K. (2003). The Trojan exosome hypothesis. (Author abstract). Proc. Natl. Acad. Sci. U. S. A. 100 (19), 10592. doi:10.1073/pnas.1831413100

Greening, D. W., Gopal, S. K., Mathias, R. A., Liu, L., Sheng, J., Zhu, H.-J., et al. (2015). Emerging roles of exosomes during epithelial-mesenchymal transition and cancer progression. Semin. Cell Dev. Biol. 40, 60-71. doi:10.1016/j.semcdb. 2015.02 .008

Griffiths, J. R. (1991). Are cancer cells acidic? Br. J. Cancer 64 (3), 425. doi:10.1038/ bjc. 1991.326

Guo, H., Wang, X., Zhu, X., Yan, M., Wang, X., Xu, Q., et al. (2019). Exosomal miR196a derived from cancer-associated fibroblasts confers cisplatin resistance in head and neck cancer through targeting CDKN1B and ING5. Genome Biol. 20 (1), 121. doi:10.1186/s13059-018-1604-0

Harvey, T. M., and Jennifer, L. G. (2005). Membrane curvature and mechanisms of dynamic cell membrane remodelling. Nature 438 (7068), 590. doi:10.1038/ nature04396

Hayden, M. S., West, A. P., and Ghosh, S. (2006). NF- $\mathrm{kB}$ and the immune response. Oncogene 25 (51), 6758-6780. doi:10.1038/sj.onc.1209943

Hazlehurst, L. A., and Dalton, W. S. (2006). De Novo and acquired resistance to antitumor alkylating agents. Totowa, NJ: Humana Press.

He, L., Ping, F., Fan, Z., Zhang, C., Deng, M., Cheng, B., et al. (2020). Salivary exosomal miR-24-3p serves as a potential detective biomarker for oral squamous cell carcinoma screening. Biomed. Pharmacother. 121, 109553. doi:10.1016/j.biopha.2019.109553

Helena Lobo, B., Rafael, L., and Jean Yj, W. (2007). DNA damage-induced cell death: lessons from the central nervous system. Cell Res. 18 (1), 17. doi:10.1038/ cr.2007.110

Hellman, N. E., and Gitlin, J. D. (2002). Ceruloplasmin metabolism and function. Annu. Rev. Nutr. 22 (1), 439-458. doi:10.1146/annurev.nutr.22.012502.114457

Holzer, A. K., Samimi, G., Katano, K., Naerdemann, W., Lin, X., Safaei, R., et al. (2004). The copper influx transporter human copper transport protein 1 regulates the uptake of cisplatin in human ovarian carcinoma cells. Mol. Pharmacol. 66 (4), 817. doi:10.1124/mol.104.001198

Hong, L., Han, Y., Zhang, Y., Zhang, H., Zhao, Q., Wu, K., et al. (2013). MicroRNA21: a therapeutic target for reversing drug resistance in cancer. Expert Opin. Therap. Targ. 17, 1073-1080. doi:10.1517/14728222.2013.819853
Iliopoulos, D., Jaeger, S. A., Hirsch, H. A., Bulyk, M. L., and Struhl, K. (2010). STAT3 activation of miR-21 and miR-181b-1 via PTEN and CYLD are part of the epigenetic switch linking inflammation to cancer. Mol. Cell 39 (4), 493-506. doi:10.1016/j.molcel.2010.07.023

Inoguchi, S., Seki, N., Chiyomaru, T., Ishihara, T., Matsushita, R., Mataki, H., et al. (2014). Tumour-suppressive microRNA-24-1 inhibits cancer cell proliferation through targeting FOXM1 in bladder cancer. FEBS (Fed. Eur. Biochem. Soc.) Lett. 588 (17), 3170-3179. doi:10.1016/j.febslet.2014.06.058

Jain, V., Das, S. N., Luthra, K., Shukla, N. K., and Ralhan, R. (1997). Differential expression of multidrug resistance gene product, P-glycoprotein, in normal, dysplastic and malignant oral mucosa in India. Int. J. Cancer 74 (1), 128. doi:10. 1002/(SICI)1097-0215(19970220)74

Jiang, F., Zhao, W., Zhou, L., Liu, Z., Li, W., and Yu, D. (2014). MiR-222 targeted PUMA to improve sensitization of UM1 cells to cisplatin. Int. J. Mol. Sci. 15 (12), 22128. doi:10.3390/ijms 151222128

Jiang, Q., Wang, Y., Hao, Y., Juan, L., Teng, M., Zhang, X., et al. (2009). miR2Disease: a manually curated database for microRNA deregulation in human disease. Nucleic Acids Res. 37 (Database issue), D98. doi:10.1093/ nar/gkn714

Johnstone, R. M. (2005). Revisiting the road to the discovery of exosomes. Blood Cells Mol. Dis. 34 (3), 214-219. doi:10.1016/j.bcmd.2005.03.002

Kernohan, M. D., Clark, J. R., Gao, K., Ebrahimi, A., and Milross, C. G. (2010). Predicting the prognosis of oral squamous cell carcinoma after first recurrence. Arch. Otolaryngol. Head Neck Surg. 136 (12), 1235-1239. doi:10.1001/archoto. 2010.214

Khoo, X. H., Paterson, I. C., Goh, B. H., and Lee, W. L. (2019). Cisplatin-resistance in oral squamous cell carcinoma: regulation by tumor cell-derived extracellular vesicles. Basel Cancers 11 (8), 144. doi:10.3390/cancers11081166

Kim, J. W., Wieckowski, E., Taylor, D. D., Reichert, T. E., Watkins, S., and Whiteside, T. L. (2005). Fas ligand-positive membranous vesicles isolated from sera of patients with oral cancer induce apoptosis of activated $\mathrm{T}$ lymphocytes. Clin. Cancer Res. Off. J. Am. Assoc. Cancer Res. 11 (3), 1010. doi:10.1034/j.1399-302x.2002.170503.x

Kim, Y. J., and Kim, J. H. (2020). Increasing incidence and improving survival of oral tongue squamous cell carcinoma. Sci. Rep. 10 (1), 7877. doi:10.1038/ s41598-020-64748-0

Kirave, P., Gondaliya, P., Kulkarni, B., Rawal, R., Garg, R., Jain, A., et al. (2020). Exosome mediated miR-155 delivery confers cisplatin chemoresistance in oral cancer cells via epithelial-mesenchymal transition. Oncotarget 11 (13), 1157-1171. doi:10.18632/oncotarget.27531

Kiyoshima, T., Yoshida, H., Wada, H., Nagata, K., Fujiwara, H., Kihara, M., et al. (2013). Chemoresistance to concanamycin A1 in human oral squamous cell carcinoma is attenuated by an HDAC inhibitor partly via suppression of Bcl-2 expression. (Research article). PloS One 8 (11), e80998. doi:10.1371/journal. pone. 0080998

Kleffel, S., and Schatton, T. (2013). Tumor dormancy and cancer stem cells: two sides of the same coin? Adv. Exp. Med. Biol. 734, 145. doi:10.1007/978-1-4614$1445-2$

Koch, R., Demant, M., Aung, T., Diering, N., Cicholas, A., Chapuy, B., et al. (2014). Populational equilibrium through exosome-mediated Wnt signaling in tumor progression of diffuse large B-cell lymphoma. Blood 123 (14), 2189. doi:10. 1182/blood-2013-08-523886

Komatsu, M., Sumizawa, T., Mutoh, M., Chen, Z. S., Terada, K., Furukawa, T., et al. (2000). Copper-transporting P-type adenosine triphosphatase (ATP7B) is associated with cisplatin resistance. Cancer Res. 60 (5), 1312. doi:10.3892/or. 8.6.1285

Kothandapani, A., Dangeti, V. S. M. N., Brown, A. R., Banze, L. A., Wang, X.-H., Sobol, R. W., et al. (2011). Novel role of base excision repair in mediating cisplatin cytotoxicity. J. Biol. Chem. 286 (16), 14564. doi:10.1074/jbc.M111. 225375

Kulkarni, B., Gondaliya, P., Kirave, P., Rawal, R., Jain, A., Garg, R., et al. (2020). Exosome-mediated delivery of miR-30a sensitize cisplatin-resistant variant of oral squamous carcinoma cells via modulating Beclin1 and Bcl2. Oncotarget 11 (20), 1832-1845. doi:10.18632/oncotarget.27557

Kuo, M., Chen, H., Song, I.-S., Savaraj, N., and Ishikawa, T. (2007). The roles of copper transporters in cisplatin resistance. Cancer Metastasis Rev. 26 (1), 71-83. doi:10.1007/s10555-007-9045-3 
Kurrey, N. K., Jalgaonkar, S. P., Joglekar, A. V., Ghanate, A. D., Chaskar, P. D., et al. (2009). Snail and slug mediate radioresistance and chemoresistance by antagonizing p53-mediated apoptosis and acquiring a stem-like phenotype in ovarian cancer cells. Stem Cells 27 (9), 2059-2068. doi:10.1002/stem.154

Larionova, I., Cherdyntseva, N., Liu, T., Patysheva, M., Rakina, M., and Kzhyshkowska, J. (2019). The role of tumor-associated macrophages (TAMs) in tumor progression. Interact. Tumor Assoc. Macroph. Cancer Chemoth. 8, e1596004. doi:10.1080/2162402x.2019.1596004

Leite, A. A., Leonel, A. C. L. d. S., Castro, J. F. L. d., Carvalho, E. J. d. A., Vargas, P. A., Kowalski, L. P., et al. (2018). Oral squamous cell carcinoma: a clinicopathological study on 194 cases in northeastern Brazil. A crosssectional retrospective study. Sao Paulo Med. J. 136, 165-169.

Li, L., Li, C., Wang, S., Wang, Z., Jiang, J., Wang, W., et al. (2016). Exosomes derived from hypoxic oral squamous cell carcinoma cells deliver miR-21 to normoxic cells to elicit a prometastatic phenotype. Cancer Res. 76 (7), 1770. doi:10.1158/0008-5472.CAN-15-1625

Li, M., Bu, X., Cai, B., Liang, P., Li, K., Qu, X., et al. (2019). Biological role of metabolic reprogramming of cancer cells during epithelial-mesenchymal transition (Review). Oncol. Rep. 41 (2), 727. doi:10.3892/or.2018.6882

Liu, T., Chen, G., Sun, D., Lei, M., Li, Y., Zhou, C., et al. (2017). Exosomes containing miR-21 transfer the characteristic of cisplatin resistance by targeting PTEN and PDCD4 in oral squamous cell carcinoma. Acta Biochim. Biophys. Sin. 49 (9), 808-816. doi:10.1093/abbs/gmx078

Liu, X., Wang, A., Heidbreder, C. E., Jiang, L., Yu, J., Kolokythas, A., et al. (2010). MicroRNA-24 targeting RNA-binding protein DND1 in tongue squamous cell carcinoma. FEBS (Fed. Eur. Biochem. Soc.) Lett. 584 (18), 4115-4120. doi:10. 1016/j.febslet.2010.08.040

Llewellyn, C. D., Linklater, K., Bell, J., Johnson, N. W., and Warnakulasuriya, S. (2004). An analysis of risk factors for oral cancer in young people: a case-control study. Oral Oncol. 40 (3), 304-313. doi:10.1016/j.oraloncology.2003.08.015

Longley, D., and Johnston, P. (2005). Molecular mechanisms of drug resistance. Chichester, UK: Springer.

Lu, W., and Kang, Y. (2019). Epithelial-mesenchymal plasticity in cancer progression and metastasis. Dev. Cell 49 (3), 361-374. doi:10.1016/j.devcel. 2019.04.010

Lu, X., and Kang, Y. (2010). Hypoxia and hypoxia-inducible factors: master regulators of metastasis. Clin. Cancer Res.: Off. J. Am. Assoc. Cancer Res. 16 (24), 5928. doi:10.1158/1078-0432.CCR-10-1360

Lv, M.-m., Zhu, X.-y., Chen, W.-x., Zhong, S.-l., Hu, Q., Ma, T.-f., et al. (2014). Exosomes mediate drug resistance transfer in MCF-7 breast cancer cells and a probable mechanism is delivery of P-glycoprotein. Tumor Markers, Tumor Targeting and Translational Cancer Research 35 (11), 10773-10779. doi:10. 1007/s13277-014-2377-z

Macfarlane, L.-A., and Murphy, R. (2010). MicroRNA: biogenesis, function and role in cancer. Curr. Genom. 11 (7), 537-561. doi:10.2174/ 138920210793175895

Manabu, M., Daisaku, Y., Hidetoshi, E., Shinichiro, H., Tomoya, K., Yoshito, T., et al. (2017). MicroRNA-155 controls exosome synthesis and promotes gemcitabine resistance in pancreatic ductal adenocarcinoma. Sci. Rep. 7 (1), 133. doi:10.1038/srep42339

Martinez-Zaguilan, R., Raghunand, N., Lynch, R. M., Bellamy, W., Martinez, G. M., Rojas, B., et al. (1999). $\mathrm{pH}$ and drug resistance. I. functional expression of plasmalemmal V-type $\mathrm{H}+$-ATPase in drug-resistant human breast carcinoma cell lines. Biochem. Pharmacol. 57 (9), 1037-1046. doi:10.1016/S0006-2952(99) 00022-2

McMahon, H. T., and Boucrot, E. (2015). Membrane curvature at a glance. J. Cell Sci. 128 (6), 1065. doi:10.1242/jcs.114454

Minciacchi, V. R., Freeman, M. R., and Di Vizio, D. (2015). Extracellular vesicles in cancer: exosomes, microvesicles and the emerging role of large oncosomes. Semin. Cell Dev. Biol. 40, 41-51. doi:10.1016/j.semcdb.2015.02.010

Ming-Hui, L., Sheng-Bo, F., and Hua-Sheng, X. (2015). Genome-wide analysis of microRNA and mRNA expression signatures in cancer. Acta Pharmacologica Sinica 36 (10), 123-129. doi:10.1038/aps.2015.67

Momen-Heravi, F., and Bala, S. (2018). Extracellular vesicles in oral squamous carcinoma carry oncogenic miRNA profile and reprogramme monocytes via NF- $\mathrm{BB}$ pathway. Oncotarget 9 (78), 34838-34854. doi:10.18632/oncotarget. 26208
Mori, K., Hiroi, M., Shimada, J., and Ohmori, Y. (2011). Infiltration of $\mathrm{m} 2$ tumorassociated macrophages in oral squamous cell carcinoma correlates with tumor malignancy. Cancers 3 (4), 3726. doi:10.3390/cancers3043726

Murakami, T., Shibuya, I., Ise, T., Chen, Z. S., Akiyama, S. I., Nakagawa, M., et al. (2001). Elevated expression of vacuolar proton pump genes and cellular ph in cisplatin resistance. Int. J. Cancer 93 (6), 869-874. doi:10.1002/ijc.1418

Mutschelknaus, L., Peters, C., Winkler, K., Yentrapalli, R., Heider, T., Atkinson, M. J., et al. (2016). Exosomes derived from squamous head and neck cancer promote cell survival after ionizing radiation. PLoS ONE 11 (10), e0152213. doi:10.1371/journal.pone.0152213

Myers, J. N., Greenberg, J. S., Mo, V., and Roberts, D. (2001). Extracapsular spread. A significant predictor of treatment failure in patients with squamous cell carcinoma of the tongue. Cancer 92 (12), 3030. doi:10.1002/10970142(20011215)92

Nath, S., Roychoudhury, S., Kling, M. J., Song, H., Biswas, P., Shukla, A., et al. (2017). The extracellular role of DNA damage repair protein APE1 in regulation of IL-6 expression. Cell. Signal. 39, 18-31. doi:10.1016/j.cellsig. 2017.07.019

Negendank, W. (1992). Studies of human tumors by MRS: a review. NMR Biomed. $5(5), 303$.

Nieto, M. A., Huang, R. Y.-J., Rebecca, A., and Thiery, J. P. (2016). Emt. Cell 166 (1), 21-45. doi:10.1016/j.cell.2016.06.028

Ogino, S., Fuchs, C. S., and Giovannucci, E. (2012). How many molecular subtypes? Implications of the unique tumor principle in personalized medicine. Expert Rev. Mol. Diagn. 12 (6), 621-628. doi:10.1586/erm.12.46

Ouyang, Y., Jiang, F., Zeng, B., Wei, C., and Yu, D. (2017). miR-222 knockdown suppresses epithelial-to-mesenchymal transitionin human oral squamous cell carcinoma. Int. J. Clin. Exp. Pathol. 10 (11), 11251-11259. doi:10.21203/rs.2. 23740/v1

O’flaherty, D. J., Barr, D. M., Fennell, D. D., Richard, D. D., Reynolds, D. J., O’leary, D. J., et al. (2012). The cancer stem-cell hypothesis: its emerging role in lung cancer Biology and its relevance for future therapy. J. Thorac. Oncol. 7 (12), 1880-1890. doi:10.1097/JTO.0b013e31826bfbc6

Pai, S., Bamodu, O. A., Lin, Y.-K., Lin, C.-S., Chu, P.-Y., Chien, M.-H., et al. (2019). CD47-SIRPa signaling induces epithelial-mesenchymal transition and cancer stemness and links to a poor prognosis in patients with oral squamous cell carcinoma. Cells 8 (12), 79. doi:10.3390/cells8121658

Pamarthy, S., Kulshrestha, A., Katara, G. K., and Beaman, K. D. (2018). The curious case of vacuolar ATPase: regulation of signaling pathways. Molecular Cancer 17 (1), 121. doi:10.1186/s12943-018-0811-3

Perez-Sayans, M., Garcia-Garcia, A., Reboiras-Lopez, M. D., and Gandara-Vila, P. (2009). Role of V-ATPases in solid tumors: importance of the subunit C (Review). Int. J. Oncol. 34 (6), 1513-1520. doi:10.3892/ijo

Pérez-Sayáns, M., Somoza-Martín, J. M., Barros-Angueira, F., Diz, P. G., Rey, J. M. G., and García-García, A. (2010). Multidrug resistance in oral squamous cell carcinoma: the role of vacuolar ATPases. Cancer Lett. 295 (2), 135-143. doi:10. 1016/j.canlet.2010.03.019

Petruzzelli, R., and Polishchuk, R. (2019). Activity and trafficking of coppertransporting ATPases in tumor development and defense against platinumbased drugs. Cells 8 (9), 139. doi:10.3390/cells8091080

Progida, C., and Bakke, O. (2016). Bidirectional traffic between the Golgi and the endosomes-machineries and regulation. J. Cell Sci. 129 (21), 3971-3982. doi: $10.1242 /$ jcs. 185702

Prohaska, J., and Gybina, A. (2004). Intracellular copper transport in Mammals1,2. J. Nutr. 134 (5), 1003-1006. doi:10.1093/jn/134.5.1003

Raghunand, N., Martinez-Zaguilan, R., Wright, S. H., and Gillies, R. J. (1999). pH and drug resistance. II. turnover of acidic vesicles and resistance to weakly basic chemotherapeutic drugs. Biochem. Pharmacol. 57 (9), 1047-1058. doi:10.1016/ S0006-2952(99)00021-0

Raposo, G., and Stoorvogel, W. (2013). Extracellular vesicles: exosomes, microvesicles, and friends. J. Cell Biol. 200 (4), 373-383. doi:10.1083/jcb. 201211138

Reichert, T. E., Strauss, L., Wagner, E. M., Gooding, W., and Whiteside, T. L. (2002). Signaling abnormalities, apoptosis, and reduced proliferation of circulating and tumor-infiltrating lymphocytes in patients with oral carcinoma. Clin. Cancer Res. Off. J. Am. Assoc. Cancer Res. 8 (10), 3137. doi:10.1093/oxfordjournals.jjco.a039559 
Ren, W., Qiang, C., Gao, L., Li, S.-M., Zhang, L.-M., Wang, X.-L., et al. (2014). Circulating microRNA-21 (MIR-21) and phosphatase and tensin homolog (PTEN) are promising novel biomarkers for detection of oral squamous cell carcinoma. Biomarkers 19 (7), 590-596. doi:10.3109/1354750X.2014.955059

Roccaro, A. M., Sacco, A., Maiso, P., Azab, A. K., Tai, Y.-T., Reagan, M., et al. (2013). BM mesenchymal stromal cell-derived exosomes facilitate multiple myeloma progression. J. Clin. Invest. 123 (4), 1542. doi:10.1172/JCI66517

Rocha, C. R. R., Silva, M. M., Quinet, A., Cabral-Neto, J. B., and Menck, C. F. M. (2018). DNA repair pathways and cisplatin resistance: an intimate relationship. Clinics 73 (Suppl. 1), e478. doi:10.6061/clinics/2018/e478ss

Roychoudhury, S., Ghuwalewala, S., Ghatak, D., Das, S., Das, P., Butti, R., et al. (2018). MiR-146a-dependent regulation of CD24/AKT/ $\beta$-catenin axis drives stem cell phenotype in oral cancer. Stem Cell Research 16 (2), 405-417. doi:10. $1101 / 429068$

Ruma Dey, G., Sangeeta, G., Pijush, D., Sapan, M., Sk Kayum, A., Jayanta, C., et al. (2016). MicroRNA profiling of cisplatin-resistant oral squamous cell carcinoma cell lines enriched with cancer-stem-cell-like and epithelial-mesenchymal transition-type features. Scientific Reports 6 (1), 111-119. doi:10.1038/ srep23932

Safaei, R., Larson, B. J., Cheng, T. C., Gibson, M. A., Otani, S., Naerdemann, W., et al. (2005). Abnormal lysosomal trafficking and enhanced exosomal export of cisplatin in drug-resistant human ovarian carcinoma cells. Mol. Cancer Therapeut. 4 (10), 1595-1604. doi:10.1158/1535-7163.Mct-05-0102

Saraiya, A. A., Li, W., and Wang, C. C. (2013). Transition of a microRNA from repressing to activating translation depending on the extent of base pairing with the target. (Research article). PLoS One 8 (2), e55672. doi:10.1371/journal.pone. 0055672

Segura, E., Nicco, C., Lombard, B., Véron, P., Raposo, G., Batteux, F., et al. (2005). ICAM-1 on exosomes from mature dendritic cells is critical for efficient naive T-cell priming. Blood 106 (1), 216. doi:10.1182/blood-2005-01-0220

Shah, M. A., and Schwartz, G. (2001). Cell cycle-mediated drug resistance: an emerging concept in cancer therapy. Clin. Cancer Res. 7 (8), 2168-2181. doi:10. 1245/aso.2004.09.909

Shields, B. (2018). "E-cadherin enhances immune control of metastatic melanoma," Editors A. J. Tackett, L. Hutchins, F. Mahmoud, S. Shalin, and H. Wong. London: ProQuest Dissertations Publishing.

Shih-Shen, L., Chih-Yu, P., Yi-Wen, L., and Cheng-Chia, Y. (2018). miR-1246 targets CCNG2 to enhance cancer stemness and chemoresistance in oral carcinomas. Cancers 7 (8), 2168. doi:10.3390/cancers10080272

Siegel, R. L., Miller, K. D., and Jemal, A. (2017). Cancer statistics, 2017. CA A Cancer J. Clin. 67 (1), 7-30. doi:10.3322/caac.21387

Sim, Y. C., Hwang, J. H., and Ahn, K. M. (2019). Overall and disease-specific survival outcomes following primary surgery for oral squamous cell carcinoma: analysis of consecutive 67 patients. J Korean Assoc Oral Maxillofac Surg 45 (2), 83-90. doi:10.5125/jkaoms.2019.45.2.83

Skog, J., Wurdinger, T., Lessard, R., Balaj, L., Carter, B., Hochberg, F., et al. (2009). Glioblastoma microvesicles transport RNA and proteins that promote tumour growth and provide diagnostic biomarkers. Cancer Res. 69, 119. doi:10.3410/f. 1129814.586895

Song, L., Yang, J., Duan, P., Xu, J., Luo, X., Luo, F., et al. (2013). MicroRNA-24 inhibits osteosarcoma cell proliferation both in vitro and in vivo by targeting LPAAT $\beta$. Arch. Biochem. Biophys. 535 (2), 128-135. doi:10.1016/j.abb.2013. 04.001

Sorenson, C. M., and Eastman, A. (1988). Mechanism of cisdiamminedichloroplatinum(H)-Induced cytotoxicity: role of G2 arrest and DNA double-strand breaks. Cancer Res. 48 (16), 4484-4488.

Specenier, B. P., and Vermorken, B. J. (2010). Advances in the systemic treatment of head and neck cancers. Curr. Opin. Oncol. 22 (3), 200-205. doi:10.1097/CCO. ob013e3283376e15

Steinbichler, T. B., Dudás, J., Skvortsov, S., Ganswindt, U., Riechelmann, H., and Skvortsova, I.-I. (2019). Therapy resistance mediated by exosomes. Mol. Cancer 18 (1), 58. doi:10.1186/s12943-019-0970-x

Tarin, D., Thompson, E. W., and Newgreen, D. F. (2005). The fallacy of epithelial mesenchymal transition in neoplasia. Cancer Res. 65 (14), 5996-6000. doi:10. 1158/0008-5472.CAN-05-0699

Théry, C., Laurence, Z., and Sebastian, A. (2002). Exosomes: composition, biogenesis and function. Nat. Rev. Immunol. 2 (8), 569. doi:10.1038/nri855
Thomas, B., Raghu, K., Nieto, M. A., and Robert, A. W. (2018). EMT in cancer. Nat. Rev. Cancer 18 (2), 128. doi:10.1038/nrc.2017.118

Trajkovic, K., Hsu, C., Chiantia, S., Rajendran, L., Wenzel, D., Wieland, F., et al. (2008). Ceramide triggers budding of exosome vesicles into multivesicular endosomes. Science (New York, N.Y.) 319 (5867), 1244. doi:10.1126/science. 1153124

Tsuzuki, H., Fujieda, S., Sunaga, H., Sugimoto, C., Tanaka, N., and Saito, H. (1998). Expression of multidrug resistance-associated protein (MRP) in head and neck squamous cell carcinoma. Cancer Lett. 126 (1), 89-95. doi:10.1016/S03043835(97)00540-5

Turchinovich, A., Weiz, L., Langheinz, A., and Burwinkel, B. (2011). Characterization of extracellular circulating microRNA. Nucl. Acids Res. 39 (16), 7223-7233. doi:10.1093/nar/gkr254

Uematsu, T., Hasegawa, T., Hiraoka, B. Y., Komatsu, F., Matsuura, T., Yamada, A. S., et al. (2001). Multidrug resistance gene 1 expression in salivary gland adenocarcinomas and oral squamous-cell carcinomas. Int. J. Cancer 92 (2), 187-194. doi:10.1002/1097-0215(200102)9999

Vega, S., Morales, A. V., Ocana, O. H., Valdes, F., Fabregat, I., and Nieto, A. M. (2004). Snail blocks the cell cycle and confers resistance to cell death. Genes Dev. 18 (10), 1134-1143. doi:10.1101/gad.294104

Wang, B., Zhang, S., Yue, K., and Wang, X.-D. (2013). The recurrence and survival of oral squamous cell carcinoma: a report of 275 cases. Chin. J. Cancer 32 (11), 614. doi:10.5732/cjc.012.10219

Wang, C., Liu, X. Q., Hou, J. S., Wang, J. N., and Huang, H. Z. (2016). Molecular mechanisms of chemoresistance in oral cancer. Chin. J. Dent. Res. 19 (1), 25-33. doi:10.3290/j.cjdr.a35694

Wang, J., Hendrix, A., Hernot, S., Lemaire, M., De Bruyne, E., Van Valckenborgh, E., et al. (2014). Bone marrow stromal cell-derived exosomes as communicators in drug resistance in multiple myeloma cells. Blood 124 (4), 555. doi:10.1182/ blood-2014-03-562439

Wei, X., Wang, W., Wang, L., Zhang, Y., Zhang, X., Chen, M., et al. (2016). MicroRNA21 induces 5-fluorouracil resistance in human pancreatic cancer cells by regulating PTEN and PDCD4. Cancer Med. 5 (4), 693-702. doi:10.1002/cam4.626

Wong, T.-S., Liu, X.-B., Wong, B. Y.-H., Ng, R. W.-M., Yuen, A. P.-W., and Wei, W. I. (2008). Mature miR-184 as potential oncogenic microRNA of squamous cell carcinoma of tongue. Clin. Cancer Res. Off. J. Am. Assoc. Cancer Res. 14 (9), 2588. doi:10.1158/1078-0432.CCR-07-0666

Wu, Y., Deng, W., and Klinke Ii, D. J. (2015). Exosomes: improved methods to characterize their morphology, RNA content, and surface protein biomarkers. Analyst 140 (19), 6631-6642. doi:10.1039/c5an00688k

Xia, Y., Shen, S., and Verma, I. M. (2014). NF-кB, an active player in human cancers. Cancer Immunol. Res. 2 (9), 823. doi:10.1158/2326-6066.CIR-140112

Xiaofeng, Z., Julienne, L. C., Jiha, K., Matthew, S., Judith, K., Hikaru, S., et al. (2015). Epithelial-to-mesenchymal transition is dispensable for metastasis but induces chemoresistance in pancreatic cancer. Nature 527 (7579), 109. doi:10. 1038/nature16064

Xu, F. H., Sharma, S., Gardner, A., Tu, Y., Raitano, A., Sawyers, C., et al. (1998). Interleukin-6-induced inhibition of multiple myeloma cell apoptosis: support for the hypothesis that protection is mediated via inhibition of the JNK/SAPK pathway. Blood 92 (1), 241.

Xuan, Z., Yu, R., Aiqin, L., Rui, J., Qingping, J., Yuanyuan, H., et al. (2014). WP1066 sensitizes oral squamous cell carcinoma cells to cisplatin by targeting STAT3/ miR-21 axis. Sci. Rep. 4 (1), 127. doi:10.1038/srep07461

Yang, S.-M., Huang, C., Li, X.-F., Yu, M.-Z., He, Y., and Li, J. (2013). miR-21 confers cisplatin resistance in gastric cancer cells by regulating PTEN. Toxicology 306, 162-168. doi:10.1016/j.tox.2013.02.014

Yoo, Y., and Kwon, Y. (2015). Molecular mechanisms controlling asymmetric and symmetric self-renewal of cancer stem cells. J. Anal. Sci. Technol. 6 (1), 1-6. doi:10.1186/s40543-015-0071-4

Yoshimura, T. (2017). The production of monocyte chemoattractant protein-1 (MCP-1)/CCL2 in tumor microenvironments. Cytokine 98, 71-78. doi:10.1016/ j.cyto.2017.02.001

Yoshizawa, K., Nozaki, S., Kitahara, H., Ohara, T., Kato, K., Kawashiri, S., et al. (2007). Copper efflux transporter (ATP7B) contributes to the acquisition of cisplatin-resistance in human oral squamous cell lines. Oncol. Rep. 18 (4), 987-991. doi:10.3892/or.18.4.987 
Yousafzai, N. A., Wang, H., Wang, Z., Zhu, Y., Zhu, L., Jin, H., et al. (2018). Exosome mediated multidrug resistance in cancer. Am. J. Cancer Res. 8 (11), 2210-2226. doi:10.1002/9780470495131.ch10

Yu, S., Wei, Y., Xu, Y., Zhang, Y., Li, J., and Zhang, J. (2016). Extracellular vesicles in breast cancer drug resistance and their clinical application. Tumour Biol. 37 (3), 2849-2861. doi:10.1007/s13277-015-4683-5

Zain, R. B. (2001). Cultural and dietary risk factors of oral cancer and precancer-a brief overview. Oral Oncol. 37, 205-210. doi:10.1016/s1368-8375(00)00133-0

Zhang, F.-F., Zhu, Y., Zhao, Q.-N., Yang, D.-T., Dong, Y.-P., Jiang, L., et al. (2014). Microvesicles mediate transfer of P-glycoprotein to paclitaxel-sensitive A2780 human ovarian cancer cells, conferring paclitaxel-resistance. Eur. J. Pharmacol. 738, 83. doi:10.1016/j.ejphar.2014.05.026

Zhang, F. F., Zhu, Y. F., Zhao, Q. N., Yang, D. T., Dong, Y. P., Jiang, L., et al. (2014). Microvesicles mediate transfer of P-glycoprotein to paclitaxel-sensitive A2780 human ovarian cancer cells, conferring paclitaxel-resistance. Eur. J. Pharmacol. 738, 83-90. doi:10.1016/j.ejphar.2014.05.026

Zhang, Y., and Wang, J. (2017). MicroRNAs are important regulators of drug resistance in colorectal cancer. Biol. Chem. 398 (8), 929-938. doi:10.1515/hsz2016-0308
Zheng, X., Li, J., Peng, C., Zhao, J., Chi, J., Meng, X., et al. (2015). MicroRNA-24 induces cisplatin resistance by targeting PTEN in human tongue squamous cell carcinoma. Oral Oncol. 51 (11), 998-1003. doi:10.1016/j.oraloncology.2015. 08.002

Zidar, N., Boštjančič, E., Malgaj, M., Gale, N., Dovšak, T., and Didanovič, V. (2018). The role of epithelial-mesenchymal transition in squamous cell carcinoma of the oral cavity. Eur. J. Pathol. 472 (2), 237-245. doi:10.1007/s00428-017-2192-1

Conflict of Interest: The authors declare that the research was conducted in the absence of any commercial or financial relationships that could be construed as a potential conflict of interest.

Copyright (C) 2021 Law, Khoo, Lim, Goh, Ming, Lee and Goh. This is an open-access article distributed under the terms of the Creative Commons Attribution License (CC $B Y)$. The use, distribution or reproduction in other forums is permitted, provided the original author(s) and the copyright owner(s) are credited and that the original publication in this journal is cited, in accordance with accepted academic practice. No use, distribution or reproduction is permitted which does not comply with these terms. 